\title{
NOTAS A LOS «RIESGOS DEL DESARROLLO»O EL «ESTADO DE LA CIENCIA» EN LA RESPONSABILIDAD CIVIL POR PRODUCTOS DEFECTUOSOS ${ }^{1}$
}

\author{
Goretti Vadillo Robredo
}

Sumario: I. Introducción. II. Concepto. III. Directiva 85/374 de la CEE. IV. El caso del Reino Unido. 1. Antecedentes. 2. Postura de la Comisión. 3. Postura del Gobierno del Reino Unido. 4. Sentencia del Tribunal de Justicia de las Comunidades Europeas de 29 de mayo 1997. V. State of the art en los Estados Unidos. 1. State of the art como cumplimiento con los «usos y costumbres de la industria». 2. State of the art como posibilidad de un diseño más seguro. 3. State of the art como riesgos conocidos pero imposibles de eliminar o riesgos desconocidos. A. Antecedentes. B. Riesgos conocidos pero imposibles de eliminar. C. Riesgos desconocidos. VI. España. 1. Antecedentes. 2. Ley 22/1994, de 6 de julio, de responsabilidad por los daños causados por productos defectuosos. 3. Análisis del art. 6.1.e). 4. Análisis del art. 6.3. Anexo. Incorporación a las leyes nacionales de los Estados miembros.

\section{Introducción}

La figura conocida como «riesgos del desarrollo» o «estado de la ciencia $»^{2}$ es probablemente una de las más polémicas y novedosas de la Ley 22/1994, de 6 de julio, de responsabilidad civil por los daños causados por productos defectuosos y en la Directiva 85/374/CEE, de 25

${ }^{1}$ La realización de este artículo ha sido posible a una beca del Gobierno Vasco (programa de becas postdoctorales de perfeccionamiento de personal investigador) disfrutada entre 1997-1998.

2 La causa de exoneración tema del presente artículo se conoce por la doctrina como «riesgos de desarrollo» (development risks, Entwicklungsgefahren, rishio di sviluppo, risque de développement) o como «estado de la ciencia» (estado de la ciencia y de la técnica, state of the art). En derecho europeo es más frecuente la utilización de «riesgos de desarrollo»y en derecho norteamericano la de state of the art. 
de julio, relativa a la aproximación de las disposiciones legales, reglamentarias y administrativas de los Estados miembros en materia de responsabilidad por los daños causados por productos defectuosos. El «estado de la ciencia» fue una de las causas principales del retraso en la aprobación de la Directiva 85/374 y también ha sido el motivo que provocó que la Comisión de las Comunidades Europeas interpusiera un recurso (con base en el artículo 169 del Tratado de la CE) contra el Reino Unido. Las cuestiones que suscitan los «riesgos de desarrollo» son de gran importancia, no sólo a nivel teórico o doctrinal, sino también a nivel práctico, ya que este tema refleja con fuerza la contraposición de intereses entre los consumidores y la industria. El problema se ha planteado, especialmente, en los productos farmacéuticos y en los de alta tecnología, de los cuales se pueden derivar efectos secundarios graves (si bien desconocidos en el momento de su puesta en circulación) que afectarán a un gran número de personas.

En Estados Unidos, por ejemplo, el conflicto se inició con el fármaco conocido como DES (Diethylstilbestrol). El DES era una hormona sintética femenina que se suministró a varios millones de mujeres entre 1945 y 1971 como tratamiento para problemas durante el embarazo, en especial como medio para evitar el aborto prematuro. Investigaciones tempranas indicaron que el DES tenía efectos tóxicos cuando se suministraba en grandes dosis. Sin embargo, cuando las dosis se reducían los efectos tóxicos, aparentemente, se eliminaban y el fármaco fue aprobado por la American Medical Association's Council on Pharmacy and Chemistry3. En 1971, los médicos del Massachusetts General Hospital de Boston informaron de un grupo de casos de adenocarcinoma vaginal en mujeres menores de 23 años ${ }^{4}$. Antes de que surgieran estos casos, el adenocarcinoma vaginal era una enfermedad casi desconocida en mujeres de dicha edad. Después de llevar a cabo un estudio médico sobre estas mujeres, se descubrió un factor común: la madre de cada una de ellas había tomado DES durante el embarazo. En 1971, la Food and

J. Solé i Feliu, El concepto de defecto del producto en la responsabilidad civil del fabricante, Valencia, 1997, pp. 478 y ss., ha dicho que aunque las dos nociones se utilizan como conceptos equivalentes, existe entre ambas un importante matiz que las diferencia. Las diferencias provienen, en nuestra opinión, de la ambigüedad que existe sobre el término state of the art en el derecho anglosajón. Sin embargo, y aunque reconozcamos la existencia de peculiaridades entre ambos conceptos, lo cierto es que la doctrina española los utiliza de forma indistinta.

3 SMITH, «Council on Pharmacy and Chemistry, Report on Council on DES», en J.A.M.A., 1942, n. ${ }^{\circ} 119$, pp. 632-635.

${ }^{4}$ Herbs, Ulfelder \& PosKanZer, «Adenocarcinoma of the Vagina», en N.Eng.J.Med., 1971 , n. ${ }^{\circ} 284$, pp. 878 y ss. 
Drug Administration (FDA) ordenó a las empresas farmacéuticas el cese en la fabricación y distribución del fármaco como método preventivo para evitar el aborto, y exigió que se informara a los médicos y al público en general de que el DES no debía ser utilizado por mujeres embarazadas dado el peligro que suponía para los fetos. Poco después de la orden de la FDA aparecieron en los tribunales los primeros casos de DES ${ }^{5}$ y en ellos surgió la pregunta de si se podía atribuir responsabilidad por un defecto imposible de descubrir según los conocimientos científicos y técnicos existentes en el momento de su puesta en circulación.

En este artículo trataremos de analizar la situación en Europa de la causa de exoneración ${ }^{6}$ de responsabilidad del «estado de la ciencia», con especial referencia a la cuestión de Gran Bretaña, la legislación española y la doctrina y jurisprudencia norteamericana.

\section{Concepto}

La Ley 22/1994, de 6 de julio, de responsabilidad civil por los daños causados por productos defectuosos, recoge en el artículo 6 las causas de exoneración de la responsabilidad, en el apartado 1.e) encontramos la causa de exoneración «riesgos del desarrollo»:

«Que el estado de los conocimientos científicos y técnicos existentes en el momento de la puesta en circulación no permitía apreciar la existencia del defecto».

La Directiva de 25 de julio de 1985, relativa a la aproximación de las disposiciones legales, reglamentarias y administrativas de los Estados

5 En los Estados Unidos otros ejemplos son la talidomida y el MER 29.

${ }^{6}$ SolÉ I Feliu, op. cit, pp. 471 y ss., utiliza la noción de «excepción» para hacer referencia a los riesgos del desarrollo. «Se trataría en este sentido, de una de las denominadas excepciones materiales, por no centrarse en la falta de requisitos o presupuestos procesales, sino en la existencia de hechos nuevos a los alegados por el demandante (no se discuten los presupuestos constitutivos del defecto del art. 3.1 LRP) que afectan al fondo del asunto (no obstante la existencia de un producto defectuoso en el sentido del art. 3.1, el art. 6.1.e) permite excluir la responsabilidad del fabricante cuando concurran los hechos en él establecidos... El hecho de que el artículo 6 LRP imponga la prueba al fabricante o importador demandados es conforme al carácter ope exceptionis y no ipso iure de las excepciones, lo que permite distinguirlas de figuras cercanas como los hechos impeditivos o los hechos extintivos». 
miembros en materia de responsabilidad por los daños causados por productos defectuosos ${ }^{7}$, en su artículo 7.e) dice:

«En aplicación de la presente Directiva, el productor no será responsable si prueba:

e) o que, en el momento en que el producto fue puesto en circulación, el estado de los conocimientos científicos y técnicos no permitía descubrir la existencia del defecto».

En 1974, el Profesor Rojo y FERnández Río no consideraba que ésta fuese una cuestión que plantease una problemática especial. En aquel entonces, parecía estar claro cuál era el problema y la solución:

«De los peligros que sean individualizables con posterioridad a la puesta en circulación como consecuencia de un avance científico o técnico (Entwicklungsgefahren, en la terminología de los autores alemanes), el fabricante no responde. En tales casos, el titular del proceso productivo podrá probar sin dificultad la ausencia de culpa.» ${ }^{8}$

La problemática de los riesgos de desarrollo surge con la aparición en Europa de las teorías del derecho norteamericano de responsabilidad objetiva y su adopción en nuestro entorno. Los riesgos de desarrollo no habían causado problemas en un sistema basado en la culpa, en el que el análisis de la conducta del fabricante es un requisito imprescindible. La dificultad se encuentra cuando se instaura un sistema, en principio, basado en la responsabilidad objetiva, en el que el análisis del comportamiento del agente pierde fuerza y deja de ser un elemento de estudio. Como dijo el Profesor Rojo y Fernández Río, aludiendo a la responsabilidad extracontractual, la referencia al nivel de conocimientos constituye el límite de la diligencia del fabricante, en cuanto que sólo responde de aquel resultado que haya previsto o que haya podido prever con arreglo al nivel de conocimientos existente ${ }^{9}$. La posibilidad de previsión hay que valorarla según el criterio recogido en el artículo 1.104 del Código Civil, es decir, en el sentido de los conocimientos científicos y técnicos que el fabricante podría alcanzar utilizando la diligencia normal en su ámbito empresarial.

En un sistema de responsabilidad que prescinde de la culpa las cosas deberían ser distintas puesto que en dicho sistema el conocimiento

7 85/374/CEE.

8 A. Rojo y FERnÁndez Río, La responsabilidad civil del fabricante, Bolonia, 1974, p. 205.

9 A. Rojo y FERnÁndez Río, op. cit., p. 204. 
del fabricante sobre el carácter defectuoso o no del producto no es relevante. Sin embargo, y como veremos a continuación, los riesgos del desarrollo fueron una de las materias más discutidas en la aprobación de la Directiva 85/374 y la causa del retraso en su adopción.

Con la expresión «riesgos del desarrollo» se hace referencia a la exoneración de responsabilidad del fabricante que pone en circulación un producto «defectuoso» siempre que en el momento de la puesta en circulación el estado de los conocimientos científicos y técnicos no permitían descubrir la existencia del defecto. La profesora PARRA LUCÁN los definió de la siguiente manera:

«... con la expresión "riesgos de desarrollo" se conoce en el ámbito de la responsabilidad de daños por productos la situación que se produce cuando el desarrollo científico y tecnológico posterior a la puesta en circulación en el mercado de su producto permite descubrir que era defectuoso y que su uso ha venido causando daños entre los consumidores» ${ }^{10}$.

De los textos legales y de las definiciones anteriores podemos extraer los requisitos para la aplicación de esta figura:

a) el defecto existe en el momento en el que el producto fue puesto en circulación.

b) el defecto no era conocido ni susceptible de ser conocido por el productor.

c) posteriormente a la puesta en circulación del producto tiene lugar el descubrimiento científico que lo revela como causa del daño.

Queda claro que la cuestión a analizar no es el carácter defectuoso o no del producto, sino el hecho de si la aparición de posteriores descubrimientos científicos o tecnológicos pueden excluir la obligación de reparar los daños que haya causado dicho producto defectuoso.

Los que defienden que el fabricante debe responder de los riesgos de desarrollo alegan que, en caso contrario todos los riesgos recaen sobre los consumidores, sin tener en cuenta que los que han obtenido los beneficios por la venta del producto han sido los fabricantes. Además, añaden que la introducción de una defensa de estas características dará lugar a que las víctimas no reciban compensación y volverá a plantear muchos de los problemas que se intentan resolver con la implantación

10 M. a A. Parra Lucán, Daños por productos y protección al consumidor, Barcelona, 1990, p. 396. 
de un sistema no basado en la culpa. Los detractores de la causa de exoneración temen que los fabricantes aleguen con frecuencia esta circunstancia, y obliguen a soportar a la víctima un proceso largo y costoso, para el que tal vez carezca de recursos económicos. Para REICH ${ }^{11}$, por ejemplo, en un sistema de responsabilidad que prescinde de la culpa es inadmisible la defensa de los riesgos de desarrollo. Por último, se afirma que son los fabricantes los que se encuentran en una mejor posición para contratar un seguro y que en el caso de que el daño se produzca son ellos los que pueden distribuir el coste a través del seguro o de un aumento en el precio de sus productos. De esta forma se conseguiría que la industria llevase a cabo mayores investigaciones y fabricase productos mejor diseñados, con mayor información y de más alta calidad.

Los que se oponen a que el fabricante responda por los riesgos del desarrollo, principalmente la industria y las compañías de seguros, aducen que no cabe que una persona espere más seguridad de un producto de la que es posible obtener de acuerdo con los conocimientos técnicos y científicos actuales ${ }^{12}$. El argumento que más peso ha tenido es el de que la imposición de responsabilidad aumentaría la carga del productor, de tal forma que se podría llegar a una paralización de las investigaciones científicas. En su opinión, no sólo perderían los fabricantes, sino que también se verían afectados los consumidores, ya que productos útiles para todos no serían puestos en circulación para evitar el riesgo de una posible responsabilidad. Además, la competitividad de las empresas afectadas por esta medida se vería disminuida por el aumento de costes frente a otros competidores que no debieran responder por los riesgos del desarrollo ${ }^{13}$. Finalmente, se plantea la cuestión del seguro. Las compañías de seguro consideran que la situación plantea una incertidumbre que escapa a toda ley de probabilidad y a toda anticipación de frecuencia, que no es medible, ni cuantificable, por lo que no es, en modo alguno, asegurable ${ }^{14}$. Se teme por el volumen y la

11 V. ReICH, «Product Safety and Product Liability - An Analysis of the EEC Council Directive of 25 july 1985 on the Approximation provisions of the Member States Concerning Liability for Defective Products», en Journal of Consumer Policy, 1986, n. ${ }^{\circ}$ 9, pp. 143-144.

12 Ver ParRa LuCán, op. cit., p. 522.

13 Frente a esta argumentación J. SolÉ I Feliu realiza una serie de interesantes reflexiones, op. cit., pp. 515 y ss.

14 Ver LEgRAND, «El impacto de la Directiva sobre el aseguramiento de la responsabilidad civil por productos defectuosos», ponencia perteneciente a la conferencia internacional sobre La Directiva 85/374/CEE sobre responsabilidad por productos defectuosos. Diez años después, Centro de Derecho de Consumo de la Universidad de Lovaina I (Bélgica), el 23 y 24 de marzo de 1995; citado en Gómez LAPLAZA, DíAz AlABART, «Responsabilidad civil por los daños causados por productos defectuosos», en Actualidad Civil, 1995, n. ${ }^{\text { }}$ 25, p. 537. 
cuantía de las posibles indemnizaciones ${ }^{15}$, que elevaría el precio del seguro.

\section{Directiva 85/374 de la CEE}

La lectura de los trabajos de elaboración de la Directiva prueba que una de las dificultades principales con que se encontraron los Estados de la Comunidad Europea, a la hora de aprobar la Directiva, fue la causa de exclusión de responsabilidad del fabricante de los riesgos del desarrollo. Se ha dicho que fue su inicial exclusión lo que provocó el retraso en la aprobación de la Propuesta de Directiva de 1976.

En 1968 comienzan los trabajos para la elaboración de la Directiva, pero éstos se ven interrumpidos por la adhesión de Gran Bretaña a la Comunidad. En agosto de 1974 se presentan dos documentos, el Memorándum de la Comisión sobre armonización de las legislaciones en materia de responsabilidad civil de productos ${ }^{16}$ y un primer Proyecto Preliminar de Directiva ${ }^{17}$. Estos trabajos darán lugar a la Propuesta de Directiva de 1976, presentada por la Comisión al Consejo de Ministros el 9 de setiembre de $1976^{18}$.

En el Proyecto de $1976^{19}$ prevaleció la opinión de excluir los riesgos del desarrollo, ya que de lo contrario se estaría haciendo una concesión injustificada al principio de responsabilidad por culpa, y esto era

15 El temor por el volumen y la cuantía de las indemnizaciones no tiene en cuenta el límite máximo de responsabilidad que se recoge en la Directiva y en la Ley 22/1994. El artículo 11 de la Ley española contempla un límite en la cuantía de 10.500.000.000 de pesetas. J. SOlÉ i Feliu piensa que es discutible que los riesgos desconocidos no puedan asegurarse: "Que en tales circunstancias las primas sean enormemente elevadas es otra cuestión, pero de ello no debe extraerse la imposibilidad de asegurar esos riesgos», op. cit., p. 509.

16 Memorandum sur le rapprochement des législations des Etats membres en matiére de responsabilité du faits des produits (Document XI/332/74-F. Document de Travail n. ${ }^{\circ} 1$ pour le Groupe de Travail «responsabilité du fait des produits»).

17 Premier avant-projet de directive sur le rapprochement des législations des Etats membres en matiére de responsabilité du faits des produits (Doc. XI/334/74-F. Document du Travail n. ${ }^{\circ} 2$ pour le Groupe de Travail «responsabilité du fait des produits», año 1974).

18 Bulletin des Communautés Européennes, supplemnt. 11/76 (Responsabilité du fait des produits).

19 Doce n. ${ }^{\circ}$ C 241, de 14 de octubre de 1976, p. 9, artículo 1.2: «El fabricante es igualmente responsable, aunque la cosa no haya podido ser considerada defectuosa en atención al desarrollo científico y tecnológico existente en el momento en que se puso en circulación». 
justamente lo que se trataba de evitar. En aquel entonces, la exclusión se consideró una victoria con concesiones hacia la minoría liderada por Alemania que logró que se estableciera un límite máximo sobre la cantidad compensatoria. En base al artículo 100.2 del Tratado de la CEE el Proyecto debía someterse a la consulta del Parlamento Europeo. El dictamen tardó tres años en salir a la luz ${ }^{20}$ y en él se recoge, entre otras ${ }^{21}$, la propuesta del Parlamento de excluir los riesgos del desarrollo. En 1979 la Comisión presenta al Consejo de Ministros una nueva Propuesta $^{22}$ en la que se mantiene la exclusión de los riesgos del desarrollo y es aquí cuando comienza el período de negociaciones que comprenderá el período de 1979 a 1985.

La Propuesta estaba claramente inspirada en el sistema norteamericano, y por ello se encontró con la oposición de los sectores industriales hacia el sistema de responsabilidad objetiva «absoluta» que en ella se contenía. La Comisión justificaba el hecho de que el productor fuese el centro de imputación ideal del daño, ya que puede «incluir los gastos que debe soportar a causa de dicha responsabilidad, como costes de fabricación, en el cálculo de los precios y repartirlos, así, entre todos los consumidores que utilizan productos del mismo tipo aunque exentos de defectos» ${ }^{23}$. Con arreglo al artículo 1 de la Propuesta, el productor:

«será responsable de los daños causados por los defectos de la cosa, prescindiendo del hecho de que los conociera. El productor será responsable asimismo si la cosa, según el estado de progreso de la técnica y la ciencia, no podía considerarse defectuosa en el momento en que la puso en circulación».

Francia, Bélgica, Dinamarca, Grecia, Irlanda y Luxemburgo eran favorables a que no se exonerara la responsabilidad del productor. Italia, Holanda y el Reino Unido mantenían la postura contraria. En 1985 se llegó a un acuerdo por el que se recogen los riesgos del desarrollo como causa de exoneración de la responsabilidad del productor (art. 7 (e.), pero se deja la posibilidad a los Estados miembros para que en su legislación no se impida la responsabilidad, artículo 15.1:

20 Doce n. ${ }^{\circ}$ C 127 , de 21-5-1979 p. 61.

21 Por ejemplo, la exclusión de los productos agrícolas, artesanales y artísticos, la liberación del fabricante que informe convenientemente al público una vez conocido el defecto, la regulación de la culpa concurrente, la reparación de los daños inmateriales y el recurso entre corresponsales sea remitida a los derechos nacionales, etc.

22 Doce n. ${ }^{\circ}$ C 271, de 26 de noviembre de 1979, p. 3.

23 Considerando quinto. 
«Cada Estado miembro podrá:

b) no obstante lo previsto en la letra e) del artículo 7, mantener o, sin perjuicio del procedimiento definitivo en el apartado 2 del presente artículo, disponer en su legislación que el productor será responsable incluso si demostrara que, en el momento en que él puso el producto en circulación, el estado de los conocimientos técnicos y científicos no permitía detectar la existencia del defecto.» ${ }^{24}$

La Directiva da la posibilidad de «mantener» la responsabilidad por riesgos del desarrollo que exista en el derecho interno de un Estado miembro, como por ejemplo la Ley alemana sobre productos farmacéuticos ${ }^{25}$. Pero además, la Directiva también prevé un procedimiento específico para el caso de que un Estado miembro quiera eliminar la causa de exoneración ${ }^{26}$. La norma comunitaria permite a los Estados miembros separarse de lo establecido en ella, no sólo en la cuestión de los riesgos del desarrollo, sino también en la ampliación del concepto de producto (art. 15.1) (permitiendo la inclusión de las materias primas agrícolas no transformadas) y en la introducción de límites cuantitativos de responsabilidad (art. 16.1 y art. 18). Para ninguna de estas otras opciones establece la Directiva un procedimiento especial. ¿Por qué

24 La explicación la encontramos en el Considerando 16 de la Directiva:

«Considerando que, por razones semejantes, ciertos Estados miembros pueden considerar una restricción injustificada de la protección del consumidor el hecho de que un productor tenga la posibilidad de liberarse de la responsabilidad si prueba que el estado de los conocimientos científicos y técnicos en el momento en que puso el producto en circulación no permitía detectar el defecto; que un Estado miembro debería, por tanto, tener la posibilidad de mantener en su legislación, o establecer en una nueva legislación, la inadmisibilidad de tal circunstancia eximente; que, en caso de una nueva legislación, el recurso a este supuesto de inaplicabilidad debe someterse a un procedimiento comunitario de mantenimiento del statu quo para elevar en lo posible el nivel de protección en toda la Comunidad de manera uniforme.»

${ }_{25}$ Conocida como Gesetzes zar Neuordnung des Arzneimittelrecht, de 24 de agosto de 1976, BGBI, 1976, I, p. 2.445.

${ }^{26}$ Artículo 15.2:

«El Estado miembro que quisiera introducir la medida especificada en la letra b) del apartado 1, deberá comunicar a la Comisión el texto de la medida propuesta. La Comisión informará de ello a los demás Estados miembros.

Este Estado miembro esperará nueve meses a tomar la medida desde el momento en que informe a la Comisión y siempre que entre tanto ésta no haya sometido al Consejo ninguna propuesta de modificación de la presente Directiva que afecte al asunto tratado. Si, no obstante, la Comisión no comunicara al Estado miembro, en el plazo de tres meses, su intención de presentar tal propuesta al Consejo, el Estado miembro podrá tomar inmediatamente la medida propuesta.

Si la Comisión presentara al Consejo la propuesta de modificar la Directiva en el mencionado plazo de nueve meses, el Estado miembro de que se trate esperará dieciocho meses para tomar la medida a partir del momento en que se presentó la propuesta». 
sólo se establece un procedimiento especial para la opción de los riesgos del desarrollo? La Exposición de Motivos lo justifica diciendo:

«...el recurso a este supuesto de inaplicabilidad debe someterse a un procedimiento comunitario de mantenimiento del statu quo para elevar en lo posible el nivel de protección en toda la Comunidad de manera uniforme».

\section{A la pregunta anterior JiMÉNEZ LIÉBANA contesta:}

«Puede no ser ajeno que éste ha sido uno de los elementos más discutidos a nivel europeo, en el que existieron posturas más enfrentadas entre la industria, consumidores y empresas aseguradoras, así como también entre distintos Estados que apoyaban distintas posiciones, que condujeron a tal situación. A la misma vez era el elemento que planteaba más difícil encaje con el sistema de responsabilidad "objetiva" que la Exposición de Motivos proclamaba. Aspecto suficientemente destacado por la doctrina, para subrayar su incompatibilidad, e, incluso, para considerar centrado el régimen de responsabilidad de la Directiva en el criterio de la culpa presunta, con lo cual ningún avance reflejaba sobre la situación existente en los países miembros, más bien un retroceso.

Parece deducirse de todo ello una voluntad política decidida a eliminar en lo posible dicha causa de exoneración, mientras lo eran en menor medida los otros supuestos, y en el momento en que se fueran dando los pasos precisos por los distintos países si hubiese sido adoptado por un número significativo de ellos. Lo prueba también el hecho de que en este supuesto, junto con la adopción del epígrafe siguiente, esté también prevista su revisión a los diez años, tal como establece el artículo15.3.»27

Tal vez el procedimiento recogido en el artículo 15 no sea más que el reflejo de un deseo por parte de los redactores de la Directiva de que, a pesar de haber llegado a un acuerdo y haber incluido los riesgos del desarrollo, con el paso del tiempo éstos serán excluidos de la Directiva y se instaurará el sistema pretendido inicialmente de responsabilidad objetiva «absoluta ${ }^{28}$ Sin embargo, el tiempo no parece haberles ayudado mucho, ya que la mayor parte de los Estados de la Unión Europea no

27 JiMÉNEZ LIÉBANA, «A propósito del "Primer informe sobre la aplicación de la Directiva en materia de responsabilidad por los daños causados por productos defectuosos (85/374/CEE)"», en Actualidad Civil, 1996, n. ${ }^{\circ}$ 26, p. 584.

${ }^{28}$ Este deseo se encuentra en la Exposición de Motivos: «si bien la armonización que resulte de la presente Directiva no puede ser total en los momentos actuales, sin embargo, abre las puertas a una mayor armonización, ...». También «(...) en esta perspectiva, es especialmente importante proceder a la revisión de aquellas disposiciones de la presente Directiva que se refieren a los supuestos de inaplicación que quedan abiertos a los estados miembros (...)». 
han hecho uso de la opción del artículo 15.1.b). Solamente en Finlan$\operatorname{dia}^{29}$ y Luxemburgo ${ }^{30}$ se responde de los riesgos de desarrollo en todos los casos. En Alemania esa responsabilidad sólo existe con respecto a los productos farmacéuticos, porque existía una ley alemana en este sentido anterior a la Directiva ${ }^{31}$. El caso de España, como luego analizaremos, es diferente porque no extiende la responsabilidad a todos los casos sino sólo a los medicamentos, alimentos o productos alimentarios destinados al consumo humano (art. 6.3).

En 1995, la Comisión elaboró el «Primer Informe sobre la aplicación de la Directiva del Consejo relativa a la aproximación de las disposiciones legales, reglamentarias y administrativas de los Estados miembros en materia de responsabilidad por los daños causados por productos defectuosos (85/374/CEE)», de acuerdo con lo dispuesto en el artículo 21 de la Directiva ${ }^{32}$. Había muchas expectativas puestas en este primer informe, pero todas ellas quedaron defraudadas, porque éste es, en primer lugar, muy breve (apenas 3 hojas). En segundo lugar, no trata la mayoría de las cuestiones que la doctrina había planteado sobre la Directiva durante estos diez años, y en tercer lugar, por el evidente retraso en su elaboración y publicación ${ }^{33}$. El Informe no hizo referencia a la situación de los riesgos del desarrollo en los Estados miembros.

\section{El caso del Reino Unido ${ }^{34}$}

\section{Antecedentes}

El 20 de setiembre de 1995, la Comisión de las Comunidades Europeas interpuso un recurso, con arreglo al artículo 169 del Tratado $\mathrm{CE}^{35}$, con objeto de que se declarase que el Reino Unido había incumplido las

29 Ley 694/90 modificada por Ley 93/93 de 8 de enero de 1993.

30 Artículo 4 de la Ley de Luxemburgo.

31 V. Ley de 24 de agosto de 1976, BGBI, 1976, I, p. 2.445.

32 Com (95) 6717 final, Bruselas, 13-12-1995. En el artículo 21 se recoge que «cada cinco años la Comisión presentará al Consejo un informe sobre la aplicación de la Directiva y, si fuera necesario, le someterá propuestas apropiadas».

${ }^{33}$ Sin embargo, tal vez el retraso en la aparición del informe no debamos achacarlo en su totalidad a la Comisión ya que sólo tres países cumplieron con su obligación de incorporar la Directiva a sus derechos nacionales dentro del plazo establecido que expiró el 30 de julio de 1988. Para más información ver JimÉnEZ LiÉBANA, op. cit., p. 559.

34 V. Sentencia del Tribunal de Justicia (Sala Quinta) de 29 de mayo de 1997, asunto C300/95.

35 En un primer momento la Comisión pensó, también, plantear este procedimiento contra Italia, aunque posteriormente sólo lo hizo contra el Reino Unido. 
obligaciones que le incumben en virtud de la Directiva 85/374/CEE y del Tratado CE, al no adoptar todas las medidas necesarias para dar cumplimiento a dicha Directiva y, en particular a la letra e) del artículo 7.

El Reino Unido ejecutó el artículo 19 de la Directiva mediante la primera parte de la Consumer Protection Act, que entró en vigor el 1 de marzo de $1988^{36}$.

La letra e) del apartado 1 del artículo 4 de la Consumer Protection Act de 1987 pretende ejecutar la letra e) del artículo 7 de la Directiva y dispone:

«En cualquier acción civil incoada al amparo de la presente parte por defecto de un producto, la parte demandada podrá exonerarse de responsabilidad si acredita (...)

e) que, en el momento relevante, el estado de los conocimientos científicos y técnicos no permitía esperar que un fabricante de productos análogos al producto de que se trate habría podido descubrir el defecto si éste hubiera existido en sus productos mientras éstos permanecían bajo su control».

La Comisión requirió al Reino Unido para que presentara observaciones sobre seis imputaciones. El Reino Unido alegó, a tenor del artículo 189 del Tratado de la CEE, que los Estados miembros tenían libertad para elegir la redacción adecuada a la hora de adaptar al Derecho interno una Directiva, siempre que se alcanzase el resultado perseguido por ésta ${ }^{37}$. El 2 de julio de 1990, la Comisión dirigió un dictamen motivado al Reino Unido. La Comisión reconocía el derecho del Estado de elegir su propia redacción para adaptar el Derecho interno a una Directiva, siempre que las disposiciones nacionales consiguieran el resultado perseguido por ésta. Sin embargo, mantuvo su postura en lo relativo a las seis imputaciones que había invocado en su escrito de requerimiento. El Reino Unido también reiteró su opinión según la cual se había adaptado el Derecho interno a la Directiva de forma correcta.

Finalmente, la Comisión desistió de todas sus imputaciones ${ }^{38}$, menos de la referida al artículo 4.1. ${ }^{\circ}$ e), al considerar que su letra carecía

$36 \mathrm{El}$ artículo 1 de la Consumer Protection Act dice:

«La presente parte tiene por objeto y efecto adoptar las disposiciones necesarias para cumplir la Directiva sobre responsabilidad derivada de los productos y deberá interpretarse en este sentido.»

37 El primer escrito de la Comisión dirigido al Reino Unido es de 26 de abril de 1989. La contestación, rechazando las alegaciones de la Comisión, es de 19 de julio de 1989.

38 Habida cuenta de la norma contenida en el apartado 1 del artículo 1 de la Consumer Protection Act, según la cual las disposiciones pertinentes debían interpretarse con arreglo a la Directiva. 
de ambigüedad y exigía de los órganos jurisdiccionales una interpretación contra legem para que fuera conforme con la Directiva. La Comisión consideraba que se había ampliado de forma considerable la excepción contenida en el artículo 7 e) de la Directiva y con ello se había convertido la responsabilidad objetiva del artículo 1 en una mera responsabilidad por culpa o negligencia. Por ello, la Comisión decidió someter al Tribunal de Justicia la compatibilidad de dicho artículo con la letra e) del artículo 7 de la Directiva.

\section{Postura de la Comisión}

Según la Comisión, el criterio sentado en la letra e) artículo 7 de la Directiva es objetivo en la medida en que hace hincapié en un estado de los conocimientos que no hace referencia alguna a la capacidad del fabricante del producto de que se trate para descubrir el defecto o a la de otro fabricante de un producto similar. Pues bien, cuando la letra e) del apartado 1 del artículo 4 de la Ley utiliza la expresión «esperar que un fabricante de productos análogos al producto de que se trate habría podido descubrir el defecto», supone una apreciación subjetiva que hace hincapié en el comportamiento de un fabricante razonable. De esta forma, sería más fácil para el fabricante de un producto defectuoso acreditar, al amparo de la letra e) del apartado 1 del artículo 4, que ni él mismo ni un fabricante de productos análogos habrían podido descubrir el defecto, en el momento relevante, dado que se respetaron las precauciones usuales en dicho sector industrial y no se incurrió en negligencia alguna, que demostrar, en los términos de la letra e) del artículo 7, que el estado de los conocimientos científicos y técnicos no permitía descubrir la existencia del defecto.

Además, la Comisión no consideraba que la referencia al artículo 1 de la Ley, aunque posee valor como indicación para los órganos nacionales, fuese suficiente para hacer legal una formulación que es contraria al texto de la Directiva.

\section{Postura del Gobierno del Reino Unido}

El Reino Unido negó que el criterio contenido en su Ley fuese un criterio «subjetivo» y que por lo tanto estableciese una responsabilidad fundada en la culpa o negligencia del agente. La referencia al «estado de los conocimientos científicos y técnicos» no alude a lo que el productor en concreto pueda saber efectivamente, sino a lo que cabría es- 
perar objetivamente de la categoría de productores a la que pertenezca, tomada en sentido genérico.

El Reino Unido recordó a la Comisión que, en cualquier caso, incumbía a sus órganos jurisdiccionales interpretar la letra e) del apartado 1 del artículo 4, de una forma compatible con la letra e) del artículo 7 de la Directiva, en virtud del artículo $1.1 .^{\circ}$ de la Ley o del principio general según el cual cualquier legislación nacional que dé cumplimiento al Derecho comunitario debe ser interpretada de conformidad con él. Consideraba que la Comisión no podía demostrar que la norma de la ley inglesa fuese incompatible con la Directiva ya que no había habido ninguna resolución de un órgano jurisdiccional acerca de esta cuestión ${ }^{39}$.

\section{Sentencia de 29-5-1997 del Tribunal de Justicia de las Comunidades Europeas}

En primer lugar, el Tribunal hizo un análisis de la disposición comunitaria. Estaba de acuerdo con la conclusión del Abogado General cuando éste aludía a los «conocimientos científicos y técnicos» y dijo que éstos se refieren, no sólo a la práctica y a las normas de seguridad usuales en el sector industrial en el que opera el productor, sino, a todo el estado de los conocimientos científicos y técnicos, incluido su nivel más avanzado existente en el momento de puesta en circulación del producto.

«27 Además, la cláusula de exoneración de la responsabilidad objeto de controversia no tiene en cuenta el estado de los conocimientos del que el productor de que se trata estaba o podía estar concreta o subjetivamente informado, sino el estado objetivo de los conocimientos científicos y técnicos del que, presumiblemente, el productor estaba informado.» ${ }^{40}$

El Tribunal rechazó el primer argumento de la Comisión según el cual el artículo $4.1 .^{\circ}$ e) de la ley inglesa utiliza un criterio subjetivo para el análisis de los conocimientos del productor.

39 En el marco de un procedimiento por incumplimiento iniciado con arreglo al artículo 169 del Tratado, corresponde a la Comisión probar la existencia del incumplimiento alegado. Es la Comisión quien debe aportar al Tribunal de Justicia los datos necesarios para que éste pueda verificar la existencia de tal incumplimiento, sin poder basarse en cualquier presunción. V. Sentencia de 20 de marzo de 1990, Comisión/Francia, C-62/89, Rec. p. I-925, apartado 27.

40 Sentencia p. I-6. 
«33 Debe rechazarse esta alegación en la medida en que hace hincapié, selectivamente, en algunas palabras de la letra e) del apartado 1 del artículo 4, sin acreditar que el contexto jurídico general, del cual forma parte la disposición controvertida, no permite garantizar efectivamente la plena aplicación de la Directiva. Pues bien, habida cuenta de este contexto, la Comisión no ha acreditado que, como pretende, el resultado perseguido por la letra e) del artículo 7 de la Directiva no se ha alcanzado manifiestamente en el ordenamiento interno.

34 En efecto, procede señalar, en primer lugar, que, conforme al artículo 7 de la Directiva, la letra e) del apartado 1 del artículo 4 de la Ley hace recaer la carga de la prueba sobre el productor que pretende invocar a su favor la cláusula exoneratoria.

35 En segundo lugar, la letra e) del apartado 1 del artículo 4 de la Ley no incluye ninguna restricción sobre el estado y el grado de los conocimientos científicos y técnicos que deben tenerse en cuenta en el momento relevante.

36 En tercer lugar, su tenor literal, considerado como tal, no permite entender que, según alega la Comisión, la aplicación de la cláusula exoneratoria depende de los conocimientos subjetivos de un productor normalmente diligente, habida cuenta de las precauciones usuales en el sector industrial de que se trate» ${ }^{41}$.

Posteriormente, analizó el segundo argumento de la Comisión basado en la interpretación que de la Ley hicieran los órganos jurisdiccionales del Reino Unido.

«37 En cuarto lugar, procede recordar que, según reiterada jurisprudencia del Tribunal de Justicia, el alcance de las disposiciones legales, reglamentarias o administrativas nacionales debe apreciarse teniendo en cuenta la interpretación que de ellas hacen los órganos jurisdiccionales nacionales (véase, en particular, la sentencia de 8 de junio de 1994, Comisión/Reino Unido, C-382/92, Rec. p. 1-2435, apartado 36). Pues bien, en el caso de autos, la Comisión no ha invocado en apoyo de su recurso resolución judicial nacional alguna que haya interpretado la disposición interna controvertida de una forma incompatible con la Directiva.

38 Finalmente, a la vista de los datos que obran en las actuaciones, nada permite considerar que las autoridades jurisdiccionales del Reino Unido, si tuvieran que interpretar la letra e) del apartado 1 del artículo 4, no lo harían a la luz de la letra y de la finalidad de la Directiva, para alcanzar el resultado perseguido por ésta y, de esta forma, atenerse al párrafo tercero del artículo 189 del tratado (véase, en particular, la

41 Sentencia pp. I-7, I-8. 
sentencia de 14 de julio de 1994, Faccini Dori, C-91/92, Rec. P. I-3325, apartado 26). Por los demás, el apartado 1 del artículo 1 de la Ley impone expresamente dicha obligación al Juez nacional.» ${ }^{42}$

El Tribunal de Justicia llegó a la conclusión de que la Comisión no había probado sus alegaciones y por lo tanto desestimó el recurso.

\section{Comentario}

La pregunta es: ¿consigue la Ley inglesa el resultado perseguido por la Directiva?

Está claro que los Estados miembros tienen libertad sobre las palabras elegidas a la hora de incorporar a sus legislaciones nacionales las Directivas. Sin embargo, el problema que se plantea en la redacción de la Consumer Protection Act de 1987 es el de si con su redacción realmente se vacía de contenido el interés de la Comunidad de establecer un sistema de responsabilidad objetiva. Si hacemos una interpretación estricta de las palabras contenidas en el artículo 4.1. ${ }^{\circ}$ e) de la Ley inglesa volveríamos al régimen en el que no sólo hay que probar el daño, la acción u omisión del agente y la relación de causalidad, si no también la culpa del agente (en este caso el productor). No es menos cierto que este régimen también recoge una inversión de la carga de la prueba; corresponde al productor demostrar que «el estado de los conocimientos científicos y técnicos no permitía esperar que un fabricante de productos análogos al producto de que se trate habría podido descubrir el defecto (...)».

La diferencia principal parece encontrarse en la introducción de la frase «un fabricante de productos análogos al producto de que se trate». Se dice que estas palabras, aunque tratan de crear un test objetivo, introducen claros elementos de subjetividad; es decir, que la Directiva se fija en el state of the art, mientras que la Consumer Protection Act descansa en la conducta del productor. CLARK se plantea ${ }^{43}$ si el elemento subjetivo de la defensa llevará a los tribunales al análisis del comportamiento de un productor medio del tipo de producto en particular ${ }^{44}$. Este autor se pregunta cuál sería el criterio del tribunal, en el caso de un productor medio, en una escala de negocios pequeña, que posee recursos limitados para la investigación y para la realización de pruebas so-

42 Sentencia p. I-8.

43 A.M. Clark, Product liability, London, 1989, pp. 153 y ss.

44 Este es el tipo de investigaciones que realiza un juez o tribunal bajo un sistema de responsabilidad basado en la culpa. V. artículo 1.104 C.c. español. 
bre sus productos. Otra dificultad que se plantea este autor es la de que puede que no existan «productores de productos análogos». De hecho, éste será el caso cuando se trate de productos nuevos.

Como hemos apuntado más arriba si los tribunales del Reino Unido hicieran una interpretación literal de las palabras del artículo 4.1.e) ello supondría una vuelta a la responsabilidad basada en la culpa del productor y así se frustraría el interés de la Comunidad en lograr una aproximación de las legislaciones de los Estados miembros en materia de responsabilidad por los daños causados por productos defectuosos.

Sin embargo, esta postura, que es la de la Comisión, olvida la regla hermenéutica del artículo primero de la Consumer Protection Act según el cual la interpretación de dicha Ley debe hacerse en el sentido de cumplir con la Directiva ${ }^{45}$. El Gobierno del Reino Unido explicó, en su Explanatory and Consultative Note ${ }^{46}$, la interpretación que debía darse a la exclusión de responsabilidad por riesgos del desarrollo:

«It is understood that the defence should be interpreted as meaning that the producer will not be liable if he proves that, given the state of scientifc and technical knowledge at the time the product was put into circulation, no producer of a product of that kind could have been expected to have discovered the existence of the defect. The burden of proof will fall squarely on the producer to show that the defect could not reasonably be expected to have been discovered. It will not necessarily be enough to show that he has done as many tests as his competitor, nor that he did an the tests required of him by a government regulation setting a minimum standard. It will therefore not be easy for a producer successfully to plead this defence...»

Al no existir resoluciones de los órganos judiciales del Reino Unido relativas a la interpretación de la Ley, el Tribunal de Justicia tuvo que pronunciarse en abstracto y de forma fundamentalmente hipotética sobre la conformidad de la referida Ley con la Directiva.

Estamos de acuerdo con el Tribunal de Justicia y con el Abogado General Sr. Giuseppe Tesauro ${ }^{47}$ en que no se puede obviar la regla del

45 Consumer Protection Act, artículo 1.1.:

«La presente parte tiene por objeto y efecto adoptar las disposiciones necesarias para cumplir la Directiva sobre responsabilidad derivada de los productos y deberá interpretarse en este sentido.»

${ }^{46}$ Implementation of the EC Directive on product Liability-An Explanatory And Consultative Note (D.I. T., november 1985), para. 22.

47 Conclusiones del Abogado General Giuseppe Tesauro presentadas el 23 de enero de 1997, asunto C-300/95, Comisión de las Comunidades Europeas contra Reino Unido de Gran Bretaña e Irlanda del Norte. 
artículo 1 de la Ley ni las obligaciones interpretativas de tenor análogo y de alcance general que impone a los jueces británicos la Europan Communities Act de 1972. La Comisión debería haber esperado a que se aplicase la Ley por los tribunales de Gran Bretaña, y entonces, si de esta aplicación se desprendía una interpretación contraria a la Directiva, entablar el procedimiento de infracción ${ }^{48}$.

\section{State of the art en los Estados Unidos}

Los antecedentes de los «riesgos del desarrollo» los encontramos en la doctrina y en la jurisprudencia norteamericana. Sin embargo, la cuestión en este país dista mucho de ser clara, por ello, a continuación haremos un pequeño análisis del state of the art/development risk en los Estados Unidos. El término utilizado es el de state of the art que tiene un significado impreciso, como consecuencia de una evolución jurisprudencial confusa y dispar entre los diferentes Estados de la Unión.

En los Estados Unidos se elaboraron por primera vez, a partir de los años sesenta, las premisas teóricas de un sistema de responsabilidad objetiva del productor. Dichas premisas fueron:

a) El mayor poder contractual y económico del productor respecto al consumidor y la función disuasoria más intensa que ejerce el régimen de responsabilidad objetiva respecto al de responsabilidad por culpa;

b) el principio del reparto de los riesgos en el seno de una determinada organización social, que debe efectuarse utilizando el mecanismo asegurador. En otras palabras, la internalización de los costes derivados de los actos perjudiciales cometidos por el productor;

c) la reducción de los denominados costes administrativos secundarios y terciarios, y la consecución de importantes ventajas sociales como consecuencia de la introducción del régimen de responsabilidad objetiva del productor.

48 De la misma opinión es el Abogado General, que en su escrito de conclusiones dice:

«... evidentemente, habría sido mucho más sensato y oportuno, antes de interponer un recurso contra el Reino Unido por la incorrecta adaptación del Derecho interno, que la Comisión hubiera esperado a la aplicación de la Ley por parte de los órganos jurisdiccionales nacionales. Y a la inversa, tal como ha sido iniciado, el procedimiento de infracción de la Comisión parece finalmente, cuanto menos, precipitado». 
Este desarrollo teórico llevó a que se aprobara la sección 402 A del Restatement (Second) of Torts en 1965, por el American Law Institute ${ }^{49}$. La historia de la responsabilidad objetiva puede dividirse en tres períodos. El primer período comprende más o menos la década posterior a la adopción del Restatement, y estuvo dominado por la pregunta de si los Estados debían adoptar la sección 402 A o no. La respuesta fue, en la gran mayoría de los casos, afirmativa. El segundo período, que transcurre durante los años setenta, enfrentó a los tribunales con una cuestión central; qué constituye un producto «defectuoso» en un sistema de responsabilidad objetiva de productos. El tercer período, en el que todavía se encuentran los Estados ${ }^{50}$, es aquel en el que los tribunales dedican gran parte de su tiempo y de sus razonamientos a los detalles de la responsabilidad objetiva, respondiendo a cuestiones como las defensas del productor, la relación de causalidad, la aplicación de la responsabilidad objetiva a situaciones o productos particulares y el state of the $\operatorname{art}^{51}$.

La cuestión de si un producto debe ser evaluado a la luz de los conocimientos científicos y de la tecnología disponible en el momento de su distribución o del proceso, en otras palabras, el análisis del state of the art, se lleva a cabo en tres ámbitos distintos. Primero, el fabricante puede fraca-

49 Restatement (Second) of Torts, 402 A (1965):

(1) One who sells any product in a defective condition unreasonably dangerous to the user or consumar or to his property is subject to liability foe physical harm thereby caused to the ultimate user or consumar, or to his property, if

(a) the seller is ingaged in the business of selling such a product and,

(b) it is expected to and does reach the user or consumer without substantial change in the condition in which it is sold.

(2) The rule stated in Subsection (1) applies although

(a) the seller has exercised all possible care in the preparation and sale of his product, and

(b) the user or consumer has not bought the product from or enterad into any contractual relation with me seller.

El antecedente directo de este texto es la conocida decisión del Tribunal Supremo de California, Greenman v. Yuba Power Products, Inc. (59.Cal.2d 57, 27 Cal.Rprt. 697. 377 P.2d 897 (1963).

${ }^{50}$ El American Law Institute está llevando a cabo durante los últimos 5 años un intento de reelaboración del Restatement (Second) en la parte correspondiente a responsabilidad por productos defectuosos. La propuesta es conocida como Restatement (Third) of Torts y no parece que vaya a tener el efecto de ruptura que supuso la adopción del Restatement (Second) of Torts, más bien parece una explicación de las cuestiones que más problemas plantean en la actualidad. En opinión de algunos autores la propuesta es un paso atrás en los derechos de los consumidores, especialmente en lo que al defecto de información y de diseño hace referencia. Por ejemplo en las secciones 1 y 2 se establece que el fabricante no será responsable por los riesgos que no se hayan podido prever de forma razonable en el momento en el que el producto se puso en el mercado.

51 Fisher \& Powers, Products Liability, St. Paul, Minn., 1994, pp. 132-133. 
sar en anticipar un accidente causado por un mal uso imprevisible del producto. Segundo, el fabricante de un producto relativamente nuevo puede no ser consciente de un riesgo genérico asociado con el producto. Tercero, el fabricante puede conocer el riesgo pero no existir la tecnología posible para reducirlo. Los tribunales norteamericanos suelen utilizar la expresión state of the art para hacer referencia al segundo y al tercer supuesto.

La expresión state of the art no se corresponde exactamente con la utilizada hasta ahora de riesgos del desarrollo (development risk) o estado de la ciencia, sino que incluye aspectos no contemplados por esta última como, por ejemplo, el hecho de que el producto posteriormente se ponga en circulación de forma más perfeccionada, y en ocasiones se ha confundido o asimilado con los «usos y costumbres de la industria». Además, el término ha tenido un tratamiento diferente según el tipo de defecto que se esté analizando en el caso en concreto: defecto de información, de fabricación o de diseño ${ }^{52}$.

Todo lo anterior nos permite ver que un análisis en profundidad del state of the art escapa a los propósitos del presente artículo, por lo que a través de algunas sentencias intentaremos aclarar las cuestiones más sobresalientes relacionadas con este tema.

1. «State of the art» como cumplimiento con los «usos y costumbres de la industria»

El primer caso en el que se utilizó el término state of the art fue Day v. Barber-Colman $\mathrm{Co}^{53}$. Una puerta móvil cayó sobre el demandante cuando la instalaba, y como resultado sufrió unas lesiones por las que demandó en base a la teoría de negligence. El demandante argumentaba que una medida de seguridad podría o debería haberse incorporado para que actuase en el momento de ajustar la puerta. El demandado presentó la prueba del state of the art, estableciendo que el producto cumplía con el patrón de diseño que era de uso común en la industria en el momento relevante y el Tribunal falló a favor del demandado:

«It is not of itself negligence to use a particular design or method in the manufacture or handling of a product... which is reasonably safe

52 DEAN WADE dijo de él que era un «chameleon-like term» y añadió que debía ser abandonado ya que «its meaning are so diverse and so often contused» (WADE, «The Effect in Product Liability of Knowledge Unavaidable Prior to Marketing», en New York University Law Review, 1983, n. ${ }^{\circ}$ 58, p. 751.; A.M. CLARK, Product Liability, London, 1989.

5310 III. App.2d. 494, 135 N.E. 2d 231 (App.Ct. 1956). 
and in customary use in the industry, although other possible designs... might be conceived which would be safer».

Sin embargo, esta prueba no es concluyente, como dijo el Juez LEARNED HAND en otro caso de negligence, en el que se consideró que existen precauciones que aunque no hayan sido tenidas en cuenta por el resto de la industria su omisión no se encuentra justificada:

«Indeed in most cases reasonable prudence is in fact common prudence; but strictly it is never its measure; a whole calling may have unduly lagged in the adoption of new and available devices... Courts must in the end say what is required; there are precautions so imperative that even their universal disregard will not justify their omission.» ${ }^{54}$

Los tribunales son unánimes en declarar que la prueba de cumplir con los «usos y costumbres de la industria» no es concluyente tampoco en un caso de responsabilidad objetiva y que por lo tanto no es una defensa para el demandado en un caso de productos defectuosos. Sin embargo, algunos han considerado que este cumplimiento sienta la presunción de que el producto no era defectuoso, tanto en un caso de responsabilidad por culpa como en uno de responsabilidad objetiva. La existencia de dicha presunción, aunque admite prueba en contrario, tiene un gran peso en contra del demandante.

Muchos tribunales admiten la prueba del state of the art en este sentido como parte de la investigación de la cualidad defectuosa del producto. Por ejemplo en Reed v. Tiffen Motor Homes Inc. ${ }^{55}$ se manifestó:

«We find that the state of the art and trade customs are relevant in helping the jury make a determination of whether the product is unreasonably dangerous when used in a manner expected by the ordinary consumer in the community. While only on element in that determination, it is necessary aid to assist the trier of fact in determinig the reasonableness of the manufacturer's design.»

Otros tribunales distinguen entre state of the art $\mathrm{y}$ las costumbres de la industria:

«State of the art does not mean the state of industry practice. State of the art means state of the industry knowledge.» ${ }^{56}$

54 The T.J. Hooper 60 F.2d. 737 (2d. Cir. 1932), p. 740.

55697 F.2d. 1192 (1983) V. CLARK, cit., p. 158.

56 V. Boatland of Houston, Inc. v. Bailey, Supreme Court of Texas, 1980. 609 S.W.2d 743. Opinión disidente. 
2. «State of the art» como posibilidad de un diseño más seguro

Un gran número de casos en los que se han planteado cuestiones del state of the art han surgido en el contexto de la posibilidad de un diseño más seguro. Estas situaciones son distintas de las recogidas en la Directiva como «estado de la ciencia». La Directiva recoge estos supuestos en el artículo 6.2 según el cual «un producto no se considera defectuoso por la única razón de que, posteriormente, se haya puesto en circulación un producto más perfeccionado».

La mayoría de los productos, con el paso del tiempo y los avances de la ciencia y de la tecnología, pueden ser diseñados de forma más segura, y esto es lo que se recoge en el artículo 6.2 de la Directiva. Parece que existe unanimidad en los Estados Unidos en que no se puede considerar que un producto está diseñado defectuosamente simplemente porque con posterioridad a su venta un avance tecnológico ha hecho posible eliminar o reducir el riesgo que dicho producto planteaba. Los tribunales han declarado casi de forma universal que la posibilidad de un producto con un diseño más seguro debe ser determinada en el momento en el que el producto fue diseñado ${ }^{57}$.

En estos casos la cuestión crucial es la de si el nuevo diseño era posible en el momento en el que el producto se puso en circulación. La prueba del demandante tenderá a demostrar que el diseño más seguro incorporado con posterioridad era posible también en el estadio anterior, con lo que el producto es defectuoso. Evidentemente, la prueba del demandado tenderá a demostrar la imposibilidad de incorporar ese diseño posterior más seguro. Ambas cuestiones estarán basadas, en gran medida, en el estado de los conocimientos científicos y tecnológicos en el momento de puesta en circulación del producto ${ }^{58}$.

Este tema hace relación a la condición defectuosa del producto y no a la causa de exclusión de responsabilidad de un producto defectuoso; en otras palabras, afecta a la determinación por el juez o el jurado de si el producto en cuestión era defectuoso.

Las teorías más utilizadas por los tribunales norteamericanos han sido:

a) El test de las «expectativas del consumidor»: se encuentra recogida en el comentario i de la sección 402 A del Restatement:

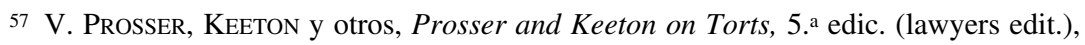
St. Paul, Minn, 1984, p. 700.

58 Estos casos deben distinguirse de aquellas situaciones en las que se argumenta que el defecto era imposible de descubrir científica o tecnológicamente. 
«The article sold must be dangerous to an extent beyond that which would be contemplated by the ordinary consumer who purchases, with the ordinary knowledge common to the community as to its characteristics».

La prueba del state of the art se encamina a establecer que si el producto es similar en diseño a otros de su misma clase el consumidor debería haber sido consciente del peligro ya que los otros productos habían sido diseñados de la misma manera y por lo tanto no podía esperar una seguridad mayor ${ }^{59}$.

Sin embargo, este test no resulta satisfactorio en muchas ocasiones porque existe gran confusión sobre si es un test objetivo o subjetivo, con lo que puede dar lugar a veredictos inconsistentes, o porque el consumidor medio puede no tener idea sobre la seguridad o peligrosidad del producto que está utilizando ${ }^{60}$.

b) El test de «risk/utility»: es uno de los métodos más utilizados para determinar el defecto del producto. En Turner v. General Motors Corp. ${ }^{61}$ se discutió la cuestión de cual debería ser el standar aplicable para determinar la condición defectuosa de un producto en un caso de responsabilidad objetiva. El Tribunal llegó a la conclusión de que la determinación de si un producto posee un defecto de diseño requiere que el jurado pondere su utilidad en contra de la probabilidad o de la gravedad de la lesión que se desprende de su uso. El jurado, continuó el Tribunal, puede considerar muchos factores antes de decidir si la utilidad o la desirability superan a los riesgos. $\mathrm{Su}$ declaración del producto como defectuoso puede estar influenciada por la prueba de un diseño más seguro que evitaría la lesión ${ }^{62}$.

59 En Bruce v. Martin-Marietta Corp (544 F.2d 442 (10 ${ }^{\text {th }}$ Cir.1976)) el Tribunal señaló:

«There is a "general" agreement that to prove liability under s.402 A the plaintiff must show that the product was dangerous beyond the expectation of the ordinary consumer. The state of the art evidence helps to determine the expectation of the ordinary consumer. A consumer would not expect a Model $\mathrm{T}$ to have the safety features which are incorporated in automobiles made today. The same expectation applies to airplanes. (The) plaintiffs have not shown that the ordinary consumer would expect a plane made in 1952 to have the safety festures of one made in 1972.»

60 V. ClaRK, cit., p. 162

61584 S.W.2d 844 (Tex. 1979).

62 Unos pocos tribunales han declarado que cuando las lesiones son graves y el producto tiene una utilidad limitada, el jurado puede declarar al fabricante responsable, aún cuando un diseño más seguro no fuese posible. V. Wilson v. Piper Aircraft Corporation, 282 Or. 61, 577 P.2d 1322(1978). En Caterpillar Tractor Co. V. Lull Eng'g Co (593 P.2d 871 (Alaska 1979)) la Corte declaró que el fact finder debe considerar como factores relevantes:

«(...) the gravity of the danger posed by the challenged desing, the likelihood that such danger would occur, the mechanical feasibility of a safer alternative design, the financial cost of an improved design, and the adverse consequences to the product and to the consumer that would result from an alternative design». 
Un elemento de gran importancia en los casos que alegan la posibilidad de un diseño alternativo más seguro es el de la determinación del momento apropiado para valorar el defecto: ¿la fecha del juicio?, ¿la fecha de distribución del producto? Hoy la mayoría de las jurisdicciones aceptan que el momento apropiado para realizar el análisis es el de la fecha de distribución del producto.

3. «State of the art» como riesgos conocidos pero imposibles de eliminar o riesgos desconocidos

Los tribunales norteamericanos también han conocido la polémica sobre si en un sistema basado en la responsabilidad objetiva el conocimiento del fabricante sobre el riesgo del producto es o no un elemento relevante en la determinación de responsabilidad y si éste puede servir como causa de exoneración de responsabilidad. Son los casos de unavoidable unsafe products. Dentro de esta categoría encontramos productos que poseen un riesgo conocido de causar daño al consumidor pero imposible de eliminar sin destruir el producto o sus propiedades esenciales y productos que poseen un riesgo sólo conocido mediante avances científicos y técnicos posteriores a su distribución.

En estos supuestos se ha distinguido ${ }^{63}$ entre:

1. Conocimiento común: Los peligros del producto son generalmente conocidos por el público, como por ejemplo el riesgo de accidente que conlleva la conducción de un automóvil o el consumo de alcohol. Salvo algunas excepciones, la mayoría de los tribunales no imponen responsabilidad en estos $\operatorname{casos}^{64}$.

2. El vendedor sabe o debería saber: Los peligros del producto no son conocidos por el público de forma general pero el vendedor los conoce o los debería poder conocer si actuase con la diligencia debida. En estos supuestos el elemento en disputa suele ser la obligación de informar que recae sobre el vendedor. Cuando el producto es un fármaco parece que una información adecuada dirigida al médico sería suficiente. Si no hay intermediario el fabricante debe avisar directamente al consumidor.

\footnotetext{
63 V. Prosser, Wade, Schwartz, Torts, Cases and Materials, 7.a edic., New York, 1982, pp. 783-784.

64 Schemel v. General Motors Corp. (7 Cir. 1967) 384 F.2d 802, en el que se declaró que el fabricante del coche no era responsable por poner en el mercado un coche que podía ser conducido a una velocidad superior de 100 millas a la hora.
} 
3. Peligros desconocidos: La existencia del peligro o del defecto es desconocida tanto para el distribuidor como para los consumidores en general. La mayoría de los casos que se han dado en los Estados Unidos han tratado con productos farmacéuticos aprobados y distribuidos por la FDA. Cuando el distribuidor no ha podido conocer el peligro, de forma general, se le exime de responsabilidad ${ }^{65}$.

\section{A. Antecedentes}

La polémica saltó con el caso Beshada v. Johns-Manville Products Corp. ${ }^{66}$ en el que algo más de 50 demandantes entablaron acciones contra los fabricantes de asbesto en base a la teoría de responsabilidad objetiva por defecto de información sobre los riesgos de la exposición al asbesto. Algunos de los demandantes habían sufrido la exposición durante los años 30. La cuestión de la apelación era si los demandados podían invocar el state of the art como defensa. Los demandados alegaban que el peligro sobre el que se debía haber informado era imposible de descubrir en el momento en el que el producto se puso en circulación dado el estado del conocimiento científico en ese momento. Se argumentaba que el conocimiento del peligro no se determinó hasta 1960, por lo que la obligación de informar no existió hasta ese momento. El Tribunal Supremo de New Jersey aceptó la alegación de que fue imposible conocer el peligro, pero abrazando una interpretación puramente doctrinal de responsabilidad objetiva, adoptó la postura de que los límites de lo científicamente posible de descubrir no afectaban a la cuestión del defecto. El Tribunal consideró que esa defensa era irrelevante en los casos de defecto de información. Entre otras cosas dijo que no aceptaba el argumento de que la teoría de risk spreading fuese inapropiada para peligros desconocidos:

«First, the same argument can be made as to hazards which are deemed scientifically knowable but of which the manufacturers were unaware... It is precisely the imputation of knowledge to the defendat that distinguishes strict liability from negligence... Second, spreading the cost of injuries among all those who produce, distribute and purchase manufactured products is far preferable to imposing it on the innocent victims who suffer illnesses and disability from defective

65 El leading case es Cochran v. Brooke (1966) 243 Or. 89, 409 P.2d 904, cuando un fármaco utilizado para el tratamiento de la artritis causó que el demandante perdiera la vista.

6690 N.J. 191, 447 A.2d 539 (1982). 
products... Finally, ...the rule will force the price of any particular products to reflect the cost of insuring against the possibility that the product will turn out to be defective.» ${ }^{67}$

Dos años después de la decisión de Beshada, el mismo Tribunal de New Jersey aceptó la defensa del state of the art. En Feldman v. Lederle Laboratories ${ }^{68}$ un caso en el que el demandante sufrió la decoloración de sus dientes por tomar tetracycline cuando era un niño. El Tribunal dijo que la prueba de que el conocimiento existente en el momento de distribución del producto no era suficiente para descubrir la existencia del defecto, y correspondía al demandado:

«Generally... available knowledge in defect warning situations (is a) relevant factor in measuring reasonableness of conduct. Generally conduct should be measured by knowledge at the time the manufacturer distributed the product. Did the defendant know, or should he have know, of the danger, given the scientifc, technological, and other information available when the product was distributed; or, in other words, did he have actual or constructive knowledge of the danger?... Under this standard negligence and strict liability in warning cases may be deemed to be functional equivalents.

In strict liability warning cases, unlike negligence cases, however, the defendant should properly bear the burden of proving that the information was not reasonably available or obtainable and that it therefore lacked actual or constructive knowledge of the defect. The defendant is in a superior position to know the technological material or data in the particular field or specialty. The defendant is the expert, often performing selftesting. It is the defendant that injected the product in the stream of commerce for its economic gain. As a matter of policy the burden of proving the status of knowledge in the field at the time of distribution is properly placed on the defendant.

(...) A warning that a product may have an unknowable danger warns one of nothing».

67 Añadió que:

«Defendants have treated the level of technological knowledge at a given time as an independent variable not affected by defendants' conduct. But this view ignores the important role of industry in product safety research. The state of the art at a given time is partly determined by how much industry invests in satety research. By imposing in manufacturers the cost of failure to discover hazards, we create an incentive for them to invest more actively in safety research»:

«In addition, discussion of the state of the art could easily confuse juries into believing that blame worthiness is at issue. Juries might mistakenly translate the confused concept of state of the art into the simple question of whether it was defendants' fault that they did not know of the hazards of asbestos.»

6897 N.J. 429. 479 A.2d 374 (1984). 
De esta forma Feldman resucitó la defensa del state of the art en los casos de defecto de información ${ }^{69}$.

No está claro cuál es el efecto final de estas sentencias dispares, ni tan siquiera en New Jersey. Puede ser que la decisión de Beshada está restringida a los productos de asbesto y la de Feldman a los farmacéuticos. Feldman parece autorizar la postura de que la prueba por el fabricante de la imposibilidad de conocer el defecto es una defensa completa en una demanda de defecto de información. CLARK considera que la postura adoptada en el caso Beshada es atractiva, pero que se le puede alegar que la doctrina de responsabilidad objetiva no es «sacrosanta» y que no debería obviar consideraciones pragmáticas ${ }^{70}$. En la actualidad se puede decir que la opinión de Beshada es claramente minoritaria en los Estados Unidos ${ }^{71}$.

\section{B. Riesgos conocidos pero imposibles de eliminar}

Una de las cuestiones más complicadas para la doctrina y la jurisprudencia norteamericana ha sido la de los productos inevitablemente inseguros, los cuales en el momento actual de conocimientos científicos y técnicos no pueden ser fabricados o distribuidos de forma segura. El termino undiscoverable risks connota un riesgo que es conocido, o sospechado, que está en el producto pero, efectivamente, tanto la presencia del peligro en un elemento particular del producto y las formas de eliminar el peligro son imposibles de descubrir. En este tema, en la gran mayoría de ocasiones se han relegado los principios de responsabilidad objetiva y se ha dado una ponderación entre la probabilidad y la gravedad del riesgo de daño frente a la utilidad del producto.

En Hines v. St. Joseph's Hospital72 la víctima recibió una transfusión de sangre del Blood Services, Inc. en el St. Joseph's Hospital, en julio

69 El Tribunal Supremo hizó mención expresa a la decisión anterior en el caso Beshada:

«If Beshada were deemed to hold generally or in all cases... that in a warning context knowledge of the unknowable is irrelevant in determinig the applicability of strict liability we would not agree. The rationale of Beshada is not applicable to this case. We do not overrule Beshada, but restrict Beshada to the circumstances giving rise to its holding.»

70 Op. cit. p. 170.

71 Algunos casos que han seguido a Beshada son:

Hayes v. Ariens Co (1984) 391 Mass 407, 462 NE2d 273, 41 ALR4th 1; Gogol v. Johns-Manville Sales Corp. (1984, DC NJ) 595 F. Supp 971; Halphen v. Johns-Manville Sales Corp. (La. 1986) 484 So.2d 110.

72 Court of Appeals of New Mexico, 1974, 86 n.m. 763, 527 P.2d 1075. 
de 1970. En setiembre comenzó un tratamiento para combatir lo que parecía una hepatitis C. Este tipo de hepatitis puede ser transmitida por un virus que infecta la sangre. La prueba en este caso estableció que existía un riesgo pequeño, pero médicamente reconocible, de que la sangre utilizada en la transfusión transmitiese a la demandante la hepatitis. Además, en el momento en el que se hizo la transfusión, no existía prueba alguna que detectase de forma adecuada la existencia del virus en la sangre y ningún proceso podía acabar con él sin destruir la sangre. Se dijo que en el momento en que se llevó a cabo la transfusión, la sangre era un producto «incapaz de proporcionar seguridad en su uso intencionado u ordinario». El Tribunal llegó a la conclusión de que las transfusiones sanguíneas no sólo eran útiles y deseables, sino que en ocasiones son esenciales para salvar la vida. Por ello, declaró que el riesgo que conllevan estaba compensado por el beneficio que aportaba su utilización al público ${ }^{73}$.

En este tema es importante tener en cuenta el comment $\mathrm{k}$ de la sección 402 A del Restatement, en donde recoge una excepción de responsabilidad para aquellos supuestos en los que se alega un defecto de diseño en base a la teoría de responsabilidad objetiva por productos defectuosos ${ }^{74}$. En realidad, no es una excepción a la responsabilidad del fabricante, sino más bien una declaración de que este tipo de productos no son defectuosos, ya que si el producto no está bien fabricado, distribuido o si la información que contiene no es suficiente nos

73 El Tribunal aplicó en este caso el test de risk/utility analizado más arriba.

$74 \ll \mathrm{k}$. Unavoidable Unsale Products. There are some products which, in the present state of human knowledge, are quite incapable of being made safe for their intended and ordinary use. These are especially common in the field of drugs. An outstanding example is the vaccine for the Pasteur treatment of rabies, which not uncommonly leads to very serious and damaging consequences when it is injected. Since the disease itself invariably leads to a dreadful death, both the marketing and the use of the vaccine are fully justified, notwithstanding the unavoidable high degree of risk which they involve. Such a product, properly prepared, and accompanied by proper directions and warning, is not defective, nor is it unreasonably dangerous. The same is true of many other drugs, vaccines, and the like, many of which for this very reason cannot legally be sold except to physicians, or under me prescription of a physician. It is also true in particular of many new or experimental drugs as to which, because of lack of time and opportunity for sufficient medical experience, there can be no assurance of safety, or perhaps even of purity of ingredients, but such experience as there is justifies the marketing and use of the drug notwithstanding a medically recognizable risk. The seller of such products, again with the qualification that they are properly prepared and marketed, and proper warning is given, where the situation calls for it, is not to be held to strict liability for unfortunate consequences attending their use, merely because he has undertaken to supply the public with an apparently useful and desirable product, attended with a known but apparently reasonable risks.» 
encontraremos ante un producto defectuoso y no se aplicará la excepción del comment $\mathrm{k}^{75}$.

La excepción del comment $\mathrm{k}$ se ha aplicado frecuentemente a las enfermedades o lesiones sufridas por una transfusión de sangre o por hemoderivados, en la que la sangre o el hemoderivado estaba contaminado. La excepción la encontramos en el caso Cunningham v. MacNeal Memorial Hospital ${ }^{76}$ en que se manifestó que la excepción k del Restatement no se aplicaba a los casos de sangre infectada, ya que ésta es «impura» y la excepción se refiere a productos con peligros inherentes pero «no defectuosos». El Tribunal declaró:

«To allow a defence to strict liability on the ground that there is no way, either practical or theoretical, for a defendant to ascertain the presence of impurities in his product would be to emasculate the doctrine (of strict liability) and in a very real sense would signal a retreat to a negligence theory.

(...) the exception relates only to products which are not impure, and which, even if properly preparad, inherently involve substantial risk of injury to the user».

Esta decisión fue muy criticada y las críticas se fundaban en que el Tribunal había ignorado la parte de la excepción en la que se contempla que en ciertos fármacos, nuevos o experimentales, en los que, por falta de tiempo o de experiencia médica, no se puede tener el convencimiento de que sean seguros o que sus ingredientes sean puros, pero que la propia experiencia médica justifica su distribución y su utilización a pesar de existir un riesgo reconocible.

Los mecanismos mediante los que los Estados niegan la indemnización para enfermedades contraídas por transfusiones de sangre (hepatitis, Sida, u otras) han sido; o excluir expresamente las transfusiones de sangre del régimen de responsabilidad objetiva, o establecer que no estamos ante un producto sino ante un servicio, de tal forma que no entra

75 El ejemplo más claro es el caso del MER/29. El fármaco MER/29 se desarrolló de forma experimental y se consideró efectivo en la reducción de colesterol en la sangre, pero cuando estaba en el mercado se descubrió que causaba cataratas y otros efectos secundarios, por lo que se retiró del mercado. Dio lugar a unas 1.500 demandas. Los primeros casos declararon que el vendedor no era responsable, en base a que el peligro era imposible de descubrir. Posteriormente, los abogados de los demandantes probaron que el fabricante era consciente de que sus pruebas no eran concluyentes ni satisfactorias y que había manipulado dichos resultados en los informes que entregó a la FDA en aras a obtener el permiso para su comercialización. V. Roginsky v. Richardson-Merrell, Inc. (1967) 251 Cal.App.2d 689, 60 Cal.Rprt. 398.

7647 III.2d 443, 266 N.E.2d 897 (1970). 
dentro del sistema del 402 A del Restatement que requiere la venta del producto para la imposición de responsabilidad objetiva.

La excepción del comment $\mathrm{k}$ del Restatement ${ }^{77}$ se ha alegado con frecuencia para los daños o lesiones causadas por el consumo de fármacos. Estos casos plantean cuestiones complicadas de resolver ya que, por ejemplo, la distribución y venta de fármacos en los USA requiere la aprobación de la agencia federal FDA. Un buen ejemplo es Grundberg v. Upjohn Co. ${ }^{78}$, en el que al Tribunal Supremo de Utah se le planteó la pregunta de si en dicho Estado se debe aplicar la excepción del comment $\mathrm{k}$ del Restatement.

El supuesto de hecho era el siguiente: Mildred Lucille Coats de 83 años murió como consecuencia de los disparos que le propinó su hija, Ilo Grundberg, el 19 de junio de 1988. Se entabla la acción y los demandantes alegan que Ilo disparó a su madre como resultado de la ingestión del fármaco Halcion, una droga fabricada para tratar el insomnio. Alegan que disparó a su madre cuando estaba bajo un estado de alucinación inducido por la droga, que conllevaba también otros efectos secundarios como depresión, psicosis, falta de personalidad, comportamiento agresivo

77 La propuesta de Restatement (Third) of Torts del American Law Institute recoge en la sección 4 la responsabilidad por daños causados por fármacos y dispositivos médicos.

La secciones más novedosas son la sección 17 y 18 en las que se recogen unas obligaciones de informar posteriores a la venta del producto.

Section 17. Liability of seller, other distributor or successor for post-sale failure to warn of risk.

One who sells or otherwise distributes a product, or who is a successor of the seller or distributor, is subject to liability for harm to persons or property caused by its failure to provide post-sale warning or instruction when:

(a) the seller or distributor (or its successor) discovers reasonably should discover, after the sale or distribution of the product, that the product poses a risk of harm to persons or property; and

(b) the persons or class of persons to whom the post-sale warning or instruction should be provided can practically be identified; and

(c) an adequate warning or instruction can practicably and effectively be communicated to, and acted on by, the identified persons or class of persons; and

(d) the risk of harm is sufficiently great that the burden of providing a post-sale warning or instruction is justified.

La sección 18 básicamente establece que el vendedor o distribuidor no serán responsables por no retirar el producto, a menos que esta retirada se requiera por ley o reglamento, o que el vendedor o distribuidor voluntariamente la realicen. La razón principal para incorporar la sección 18 es la de dejar claro que entre las obligaciones de la sección 17 no se encuentra la de retirar el producto.

Sin embargo, hay que tener en cuenta que aún en el caso, de que esta propuesta se aprobase finalmente, habría que esperar unos años para ver cuál es su aceptación en los tribunales y en los Estados, ya que el ALI carece de poder legislativo.

78 Supreme Court of Utah, 1991, 813 P.2d. 89. 
y homicida. Alegan, entre otras cuestiones, que el fabricante fracasó en informar sobre los efectos secundarios de la droga y que además, ésta posee un defecto de diseño. Los demandados argumentaron que el orden público apoya la idea de que las investigaciones y el desarrollo de nuevas drogas requieren la declaración de que todos los medicamentos aprobados por la FDA son unavoidably unsafe products bajo el comment $\mathrm{k}$ y por lo tanto los fabricantes de esas drogas no deben ser declarados responsables en una demanda basada en defecto de diseño. Los demandantes alegaron que la excepción del comment $\mathrm{k}$ no podía ser aplicada de forma general para todos los fármacos sino que se debe determinar si cada fármaco en particular está o no dentro del ámbito del comment $\mathrm{k}$.

El Tribunal ${ }^{79}$ estuvo de acuerdo con la proposición básica del comment $\mathrm{k}$, es decir, que existen ciertos productos que poseen peligros asociados con su uso aún cuando se utilizasen la forma adecuada para ellos. Estaban de acuerdo también con que el vendedor de esos productos - cuando el producto está preparado adecuadamente y distribuido con la información adecuada - no debe ser declarado responsable en base a la teoría de responsabilidad objetiva por las «consecuencias desafortunadas» que provoque su uso. Por ello, adoptan el comment k como derecho aplicable en Utah, y analizan la cuestión de cómo debe ser aplicado.

El Tribunal aclaró que la inmunidad del comment $\mathrm{k}$ sólo existe en los casos de defecto de diseño, y que no se extiende al defecto de información o al de fabricación, puesto que su propósito es el de proteger a aquellos productos que no pueden ser diseñados de forma más segura. Esta limitación en el ámbito de aplicación del comment $\mathrm{k}$ se acepta universalmente. A continuación analiza la polémica de si la aplicación del comment $\mathrm{k}$ debe hacerse de forma general para todos los fármacos o si ésta requiere un análisis case by case, y para ello se remite a la jurisprudencia.

California fue el primer Estado en aplicar el test de risk/benefit para determinar qué drogas tienen derecho a la protección del comment $\mathrm{k}$, en Kearl v. Lederle Laboratories ${ }^{80}$. El Tribunal de Kearl trató los problemas

79 El Tribunal comenzó su exposición analizando la ley aplicable en el Estado de Utah. En primer lugar reconocen que en dicho Estado se aprobó la sección 402 A del Restatement en Ernerst W. Hahn, Inc. v. Armco Steel Co. (601 P.2d 152, 158 (Utah 1979)), y por lo tanto también el comment $\mathrm{k}$.

80172 Cal.App.3d 812, 218 cal.Rptr 453 (1985). Este caso fue anulado luego en Brown v. Superior Court (44 Cal.3d 1049, 245 Cal.Rprt, 412, 751 P.2d 470, (1988)), en el que se estableció que la norma en California es que todos los fármacos con prescripción médica tienen derecho a la protección del comment $\mathrm{k}$. 
que se causan en la investigación y en el desarrollo de nuevas drogas y expresó su desacuerdo con el método «mecánico» por el que muchos tribunales de apelación concluían que los fármacos tienen derecho a este tratamiento especial. En su opinión, la decisión debía tomarse en base al test de risk/benefit. Bajo este test un producto estará dentro del ámbito de aplicación del comment $\mathrm{k}$ sólo si el tribunal llega a la conclusión de que: (1) el producto pretendía lograr un beneficio excepcionalmente importante, (2) el riesgo que poseía era inherente e imposible de evitar cuando se distribuyó.

En contraste con estos tribunales que aplicaron la excepción en base a un case by case se encuentran aquellos tribunales que apoyan la postura de que todas las drogas prescritas tienen derecho a esta excepción. En Brown v. Superior Court el Tribunal trató un caso en el que se demandaba a los fabricantes de diethystibestrol. El Tribunal consideró que el test de risk/benefit suponía un procedimiento impracticable por su impacto negativo en el desarrollo de nuevos fármacos. Además consideró que era demasiado amplio y que daría lugar a que jueces distintos resolvieran casos iguales de forma diferente. El Tribunal valoró tres cuestiones para no imponer responsabilidad objetiva en estos casos. Primero, los fabricantes podrían parar su produción de drogas valiosas para la comunidad por la pérdida de beneficios resultantes de las demandas y por la incapacidad de conseguir un seguro adecuado. Segundo, los consumidores tienen interés en disponer rápidamente de los nuevos productos farmacéuticos. Imponer responsabilidad por defecto de diseño puede causar que los fabricantes retrasen la salida de nuevos productos al mercado, aun cuando tengan la aprobación de la FDA. Tercero, el coste añadido de asegurarse en contra de la responsabilidad objetiva y los programas adicionales de investigación puede llevar a que el coste de la medicación aumente a un punto en el que los consumidores no puedan comprarlo. Concluyó que la excepción era aplicable en base al interés público en el desarrollo y en el precio razonable de fármacos.

El Tribunal Supremo en Grudberg, después del estudio de la jurisprudencia de otros Estados, decidió apoyar la decisión de Brown, según la cual el método del case by case no funcionaba correctamente y la excepción debía aplicarse a todos los fármacos de forma general:

«We agree with the principle comment $\mathrm{k}$ embodies, that manufacters of unavoidably dangerous products should not be liable for a claim of desing defect. We are persuaded that all prescription drugs should be clasified as unavoidaby dangerous in desing because of their unique nature and value, the elaborate regulatory systems overseen by the FDA, 
the difficulties of relying on individual lawsuits as a forum in which to review a prescription drug's desing, and the significant public policy considerations notad in Brown. We therefore reach the same conclusion as did the California Supreme Court in Brown, albeit pursuant to a slightly different rationale».

También analizó las características únicas de los productos farmacéuticos, que a la postre es la razón que les llevó a tomar la decisión anterior $^{81}$. El Tribunal no olvidó en su análisis a la FDA ${ }^{82}$. Como consecuencia de todo lo anterior el Tribunal Supremo dijo:

«We find this extensive regulatory scheme capable of and appropriate for making the preliminary determination regarding whether a prescription drug's benefits outweigh its risks. The structured follow-up program imposed by law ensures that drugs are not placed on the market without continued monitoring for adverse consequences that would rendar the FDA's inicial risk/benefit analysis invalid. Allowing individual courts and/or juries to continually reevaluate a drug's risks and benefits ignores the processes of this expert regulatory body and the other avenues of recovery available to plaintiffs».

La respuesta final del Tribunal de Utah fue contraria a las pretensiones de los demandantes, aunque les reconoció que disponían de otras vías:

«In light of the strong public interest in the availability and affordability of prescription medications, the extensive regulatory system of the FDA, and the avenues of recovery still available to plaintiffs by claiming inadequate warning, mismanufacture, improper marketing, or misrepresenting information to the FDA, we conclude that a broad grant of immunity from

81 «Despite inherent risks, and in contrast to any other product, society has determined that prescription medications provide a unique benefit and so should be available to physicians with appropriate warnings and guidance as to use. The federal government has established an elaborate regulatory system, overseen by the FDA, to control the approval and distribution of these drugs. No other class of products is subject to such special restrictions or protections in our society.»

82 United States v. Sullivan, 332 U.S. 689, 696, 68 S.Ct. 331, 335, 92 L.De. 297 (1948). La agencia se creó para proteger a los consumidores de los poductos peligrosos. Antes de la aprobación de un medicamento nuevo la FDA utiliza importantes mecanismos para asegurar que los beneficios potenciales del producto superan a sus riesgos asociados. Este proceso puede requerir años de pruebas y exámenes. Pero además, la FDA también lleva a cabo investigaciones una vez que el producto sale al mercado. Todos los informes sobre reacciones adversas de un fármaco deben ser comunicados a la FDA por médicos, investigadores o fabricantes. 
strict liability claims based on desing defects should be extended to FDAapproved prescription drugs in Utah.» ${ }^{83}$

Como conclusión cabe decir que del análisis de la jurisprudencia norteamericana se desprende que en la aplicación de la excepción del comment $\mathrm{k}$ no existe unanimidad. Algunos tribunales evalúan cada producto para determinar si éste es unavoidable unsafe, mientras que otros, como el Tribunal de Utah, declaran que todos los productos de una categoría (en concreto, todos los productos farmacéuticos) son unavoidable unsafe.

\section{Riesgos desconocidos}

El último grupo de casos a los que se aplica el state of the art son aquellos en los que el producto contiene un peligro o un riesgo de causar daños que resulta desconocido en el momento en el que se pone en

83 En este caso hubo una opinión disidente, la del Juez STEWARD, que recogemos a continuación de forma parcial:

«In truth, FDA safety procedures do not justify abdication of judicial responsability. For example, the FDA does not required existing drugs to undergo newly development tests which would increase the likelihood that a product is in fact safe (...)

(...) Numerous congressional investigations have demostrated that the FDA has often approved drugs in complete ignorance of critical information relating to the hazard of such drugs which was contained either in its own files or in the published medical literature, or both.

In relying on the efficacy of FDA approval procedures as the basis for dispensing with the judicial remedy of product liability, the majority simply ignores FDA failures to protect the public against unnecessary ans unacceptable risks (...)

Furthermore, not a shred of evidence has been presentad to this Court that inidicates that liability under the tort system has deterred pharmaceutical companies from introducing new drugs. Even if that were the case, the question that must be answered, given the majoritiy's holding, is why comment $\mathrm{k}$ does not provide a proper accomodation of all the competing policy interests involved in the issue before the Court. Why should those who are seriously injured or suffer because of the death of another have to stand the expense of such losses to support the high profit margins inthe drug industry?

The majority opinion states that a case-by-case analysis would leave drug companies uncertain regarding questions of immunity and would result in patchwork verdicts when a drug may be found to be subject to comment $\mathrm{k}$ exemption in one case but not suject to the exemption in another case. That consideration has little merit, in my view. We tolerate nonuniformity of results in negligence cases at all time. Nothing this Court does can bring about uniformity of result with respect to drugs. The states are already divided on the issue of whether FDA approval of a drug should confer immunity from design defect, although it apperas that no state has gone as fer as Utah now does. Suffice to say, a number of courts apply comment $\mathrm{k}$ on a case-by-case basis - a task that cannot be avoided even under the majority's position if a strict liability claim is coupled with a negligence claim, as is usually the case.» 
circulación en el mercado ${ }^{84}$ y sólo con posterioridad y gracias a los avances de la ciencia y de la tecnología se descubre la cualidad defectuosa del producto. Al igual que en los otros supuestos que hemos tratado hasta el momento, también aquí la jurisprudencia norteamericana se encuentra dividida. Existen tribunales que consideran que el desconocimiento del riesgo es irrelevante bajo la teoría de responsabilidad objetiva (el leading case es Beshada v. Johns-Manville Products Corp.) ${ }^{85}$. Aunque ésta es la postura minoritaria, ha sido adoptada, por ejemplo, por el Tribunal Supremo de Missouri, en Elmore v. Owens-Illinois ${ }^{86}$.

Sin embargo, la mayoría de las jurisdicciones han seguido la postura de Feldman v. Lederle Laboratiries ${ }^{87}$ y la incapacidad científica de descubrir el defecto funciona como causa de exoneración de responsabilidad del demandado, que es sobre quien recae la carga de la prueba.

En los Estados Unidos el problema surgió con fuerza en los daños causados por el asbesto. Un buen ejemplo es Owens-Illinois, Inc. v. Zenobia ${ }^{88}$, en el que el Tribunal analizó la aplicación del state of the art en un caso de responsabilidad objetiva frente a los que se resuelvan bajo la teoría de negligencia.

El punto de partida es la sección 402 A del Restatement, tantas veces citada en este texto, según la que el demandado debe probar:

«(1) [that] the product was in a defective condition at the time it left the possession or control of the seller, (2) that it was unreasonably dangerous to the user or consumer, (3) that the defect was a cause of the injuries, and (4) that the product was expected to and did reach the consumer without substantial change in its condition».

Por lo tanto, la sección 402 impone responsabilidad sin tener en cuenta el conocimiento del defecto y aunque el vendedor haya puesto en práctica todas las medidas de cuidado posible en la preparación y venta del producto ${ }^{89}$. Cuando se ha alegado que un producto es defectuoso por

${ }^{84}$ Este es el que se corresponde con la cláusula de exclusión de responsabilidad de la Directiva 85/374 y de la Ley 22/1994.

8590 N.J. 191, 447 A.2d 539 (1982).

86673 S.W.2d. 434 (Mo. 1984). El Tribunal Supremo consideró irrelevante el hecho de que el fabricante no pudiese haber conocido los riesgos de su producto porque en Missouri el test que se aplica es el de las «expectativas del consumidor» de la sección 402 A del Restatement, y su aplicación junto con los fines de la responsabilidad objetiva hacen que la incapacidad de descubrir el riesgo no pueda ser considerada una defensa para el demandado.

8797 N.J. 429, 479 A.2d. 374 (1984).

88 Court of Appeals of Maryland, 1992, 325 MD 420, 601 A.2d 633.

89 V. Restatement (Second) of Torts 402 A, comment a (1965). 
defecto de información muchos tribunales han considerado aplicable el comment $\mathrm{j}$ del Restatement ${ }^{90}$ en el que se distingue el producto que contiene una información adecuada del producto defectuoso:

«a product bearing such a warning, which is safe for use if it is followed, is not in defective condition, nor is it unreasonably dangerous».

Algunos tribunales han reconocido que este comentario parece contradecir o crear una excepción a la regla de la sección 402 A. Pero la mayoría de los tribunales que han considerado el defecto de información bajo la teoría de strict liability han declarado expresa o implícitamente que el fabricante de un producto, que es defectuoso sólo por la falta de información adecuada, no es responsable cuando el defecto de información resulte de la ausencia de conocimiento de la cualidad peligrosa del producto. Además, alegan que la existencia del conocimiento en el demandado puede ser establecido mediante la prueba de que la cualidad peligrosa del producto debería haber sido conocida por el fabricante porque era conocida en la comunidad científica o de expertos. El Juez Minor WisDom, en otro caso de asbesto, en relación al conocimiento que debe tener el fabricante dijo ${ }^{91}$ :

«in cases such as the instant case, the manufacter is held to the knowledge and skill of an expert. This is relevant in determinig (1) whether the manufacter knew or should have know the danger... The manufacter's status as expert means that at the minimum he must keep abreast of scientific knowledge, discoveries, and advances and is presumed to know what is imparted therby».

La mayoría de los tribunales norteamericanos manifiestan que la prueba del state of the art es pertinente en los casos de defecto de información ${ }^{92}$. La Corte de Apelación de Maryland, en el caso Zenobia,

90 Comment j: «the seller is required to give warning against "the danger", if he has knowledge, or by the application of reasonable, development human skill and foresight should have knowledge, of the (...) danger (...)».

91 Borel v. Fibreboard Paper Products Corporation, 493 F.2d 1076, 1089 (5 $5^{\text {th }}$ Cir.1973), 419 U.S. 869, 95 S.Ct. 127, 42 L.De.2d 107 (1974).

92 Los profesores HENDERSON y TWERSKI argumentan que la diferencia entre strict liability y negligence en un caso de defecto de información es semántica e innecesariamente confusa. (V. Henderson, TwersKI, «Doctrinal Collapse in Products Liability: The Empty Shell of Failure to Warn», en New York University Law Review, 1990, n. ${ }^{\circ} 65$, p. 265). 
reconoció que en el defecto de información del Restatement se han colado conceptos de negligencia. En el caso juzgado ante la Corte de Maryland los demandantes alegaron que la prueba sobre el conocimiento del fabricante no debería ser relevante. Los demandados alegaron que la otra parte debería probar el state of the art como parte de su caso. El trial judge estuvo de acuerdo con estos últimos y requirió a los demandantes para que probaran el state of the art. El United States Court of Appeals aplicando la ley de Maryland declaró que en estos casos la prueba es relevante en relación a la responsabilidad del demandado. La Corte de Apelación de Maryland llegó a la conclusión, después del análisis de la jurisprudencia del Estado, de que el vendedor (o fabricante) no puede ser declarado responsable bajo la teoría de responsabilidad objetiva a menos que conociera el peligro o lo debiera haber conocido. La imposibilidad técnica o científica de conocer el defecto o el peligro inherente del producto funciona como causa de exención de responsabilidad. El análisis del conocimiento del agente debe ser evaluado, según manifestó el Tribunal, en base al conocimiento que posee un experto de su misma área ${ }^{93}$.

Lo que no queda claro es si la prueba de la posibilidad o imposibilidad de conocer la existencia del defecto recae sobre el demandante o sobre el demandado. Los profesores Prosser y KEETON son de la opinión de que la carga de la prueba debe recaer sobre el demandante ${ }^{94}$. Sin embargo, en un gran número de sentencias se ha declarado que la carga de la prueba recae sobre el demandado, ya que el state of the art funciona como causa de exclusión de responsabilidad. En Feldman v. Lederle Laboratories ${ }^{95}$ el Tribunal dijo que en los casos de defecto de información bajo la teoría de responsabilidad objetiva, a diferencia de aquéllos que se entablen bajo la teoría de negligencia, sobre el demandado recae la carga de la prueba de que no existía información sobre la existencia del defecto y que, por lo tanto, carecía de conocimiento alguno sobre la existencia del defecto. La razón esgrimida fue la de que el demandado se encuentra en una posición superior para conocer los

93 Las palabras del Tribunal fueron:

«We hold that Comment j of 402 a is applicable to a strict liability cause of action where the alleged defect is a failure to give adequate warnings. Therefore, the seller is not strictly liable for failure to warn unless the seller has "knowledge, or by the application of reasonable, development human skill and foresight should have knowledge, of the presence of the... danger". Restatement (Second) of Torts 402 A, Comment j. Moreover, we agree with the numerous cases holding that, for purposes of the "should have knowledge" component of comment $\mathrm{j}$, a manufacturer of a product is held to the knowledge of an expert in the field.»

94 Prosser, Prosser and Keeton on Torts, cit., p. 697.

9597 N.J. 429, 479 A.2d 374(1984). 
materiales o las informaciones tecnológicas en un ámbito determinado. Además, que es el demandado el que ha introducido el producto en el mercado para su beneficio económico ${ }^{96}$.

\section{España}

\section{Antecedentes}

Hasta 1984, el sistema legal español se basaba en las provisiones de los códigos decimonónicos (Código Civil y Código de Comercio) que establecen una responsabilidad predominantemente basada en la culpa o negligencia del agente. Nuestra legislación se acercó a un sistema basado en la responsabilidad objetiva en la Ley General para la Defensa de los consumidores y usuarios de 1984 (L.C.U.) ${ }^{97}$. En el artículo 26 de la L.C.U. se contempla un régimen de responsabilidad por culpa con inversión de la carga de la prueba que permite al demandado liberarse de responsabilidad si «consta o se acredita que se han cumplido debidamente las exigencias y requisitos reglamentarios establecidos y los demás cuidados y diligencias que exige la naturaleza del producto, servicio o actividad» ${ }^{98}$. Bajo el artículo 26 el demandado podrá exonerarse de responsabilidad si prueba que se ha ajustado a las técnicas habituales de producción del sector, que empleó los materiales utilizados en otras empresas, etc. Es decir, no se encontrará culpa alguna en el demandado simplemente por ignorar lo que nadie en ese momento conocía.

96 Aunque en el caso de Maryland no se planteó la cuestión, la Corte dijo que la carga de la pueba recaía sobre el demandante.

97 Ley 26/1984, de 19 de julio. El origen de la Ley de Consumidores de 1984 fue la conmoción social provocada por el envenenamiento masivo producido por el aceite de colza desnaturalizado.

98 Vid. PARRA LuCÁn, Daños por productos y protección al consumidor, op. cit., pp. 376 y ss:

«De la lectura del precepto se desprende que en el artículo 26 el legislador quiso aplicar a los daños por productos la formulación jurisprudencial del artículo 1.902.»

La opinión contraria fue la adoptada por BERCOVITZ RODRÍGUEZ-CANO, «La adaptación del derecho español a la directiva comunitaria sobre responsabilidad por los daños causados por productos defectuosos», Estudios sobre Consumo, n. ${ }^{\circ}$ 12, abril 1988, p. 117 :

«Parece lógico entender que nuestro legislador ignoró el tema. Consecuentemente parece lógico y prudente deducir que no pretendió extender su protección a los mencionados riesgos... De acuerdo con lo dicho, nuestra LGDCU no incluye la responsabilidad por este tipo de riesgos... La falta de mención de esta cuestión en la LGDCU equivale, pues, a un rechazo de la inclusión de semejante responsabilidad a cargo de los productores y fabricantes.» 
En el artículo 28 de la L.C.U. se encuentra la primera aproximación de nuestro sistema, en materia de responsabilidad por productos, a la responsabilidad objetiva y no se recoge, en dicho artículo, ninguna excepción. Parece entonces que la excepción de los riesgos del desarrollo no fue contemplada por el legislador de $1984{ }^{99}$ y que en este artículo rige la responsabilidad por los riesgos de desarrollo. La profesora PARRA LuCÁN, en búsqueda de una solución intermedia, respetuosa con el tenor del artículo 28 y con los intereses de los consumidores y los productores, analiza el supuesto de una vacuna que cura cierta enfermedad aunque provoca efectos secundarios graves, por ejemplo cuando la víctima queda ciega pero, de no haber sido vacunada, hubiera muerto. PARRA LUCÁn no se cuestiona (como sí se ha hecho en los Estados Unidos en el análisis del risk/benefit) que el producto no sea defectuoso; su pregunta va encaminada a si la reparación debe o no ser aminorada, compensando el daño con los beneficios obtenidos por el producto:

«Así, habría de entender que no hay daño causado sino en lo que éste supere a la ventaja proporcionada. En definitiva la aceptación de esta tesis supondrá que el consumidor no podrá obtener la reparación de ciertos daños si a causa del uso o consumo del producto que se los ha originado le han sobrevenido ciertas consecuencias beneficiosas. Desde luego que la consideración de las ventajas obtenidas para disminuir la reparación ha de tener en cuenta otras circunstancias tales como la imposibilidad de lograr por otra vía el beneficio obtenido.»100

\section{Ley 22/1994, de 6 de julio, de responsabilidad civil por los daños causados por productos defectuosos ${ }^{101}$}

En el largo proceso legislativo de la nueva Ley, se partió de un primer borrador del profesor RoJo, sobre cuya base la Comisión General

99 V. PARRA LuCAN, p. 397:

«En efecto, con la lectura de los debates parlamentarios se comprueba que el legislador de 1984 ignoraba los problemas que podía plantear la evolución de los conocimientos científicos y técnicos acerca del carácter defectuoso de un producto.» Esta autora también recoge la enmienda n. ${ }^{\circ} 115$ presentada por Escuder Croft al artículo 27 del Proyecto de Ley de 2 de diciembre de 1983, proponiendo una nueva redacción. El artículo 27.3.a) propuesto establecía:

«3. La responsabilidad del fabricante podrá ser suprimida o reducida por los Tribunales en aquellos supuestos que a continuación se indican:

a) Que el defecto fuese debido a caso fortuito. El fabricante no responde de aquellos daños que no hubiera podido prever o que previstos fueran inevitables.»

100 PARRA LuCÁN, cit., p. 399.

101 Ley 6-7-1994, n. ${ }^{\circ}$ 22/1994. 
de Codificación reelaboró un anteproyecto. Posteriormente, el 12 de febrero de 1992, el Gobierno aprobó un Anteproyecto de Ley, que el adelanto de las elecciones generales truncó, dando lugar al de 20 de diciembre del mismo año y finalmente a la Ley 22/1994, de 6 de julio.

Una de las diferencias que existen entre la Ley española y la Directiva se encuentra en el artículo 6.3 (en donde se ha hecho uso de la posibilidad prevista en el artículo 15.1.b):

Artículo 6. Causas de exoneración de la responsabilidad.

1. e) Que el estado de los conocimientos científicos y técnicos existentes en el momento de la puesta en circulación no permitía apreciar la existencia del defecto.

3. En el caso de medicamentos, alimentos o productos alimentarios destinados al consumo humano, los sujetos responsables, de acuerdo con esta Ley, no podrán invocar la causa de exoneración de la letra e) del apartado 1 de este artículo.

El artículo 6.3 de la Propuesta de Anteproyecto de la Ley de responsabilidad civil por los daños causados por productos defectuosos recogía la responsabilidad por los riesgos del desarrollo para los fabricantes de medicamentos destinados al consumo humano ${ }^{102}$. La inclusión de los alimentos se produjo en el Anteproyecto del Ministerio de Justicia de ley de responsabilidad por daños causados por productos defectuosos ${ }^{103}$, y apareció después en los sucesivos proyectos de febrero y diciembre de $1993^{104}$.

La adopción del artículo 6.3 ha sido llamativa para ciertos sectores doctrinales. Por ejemplo, Gómez LaPlaza y Díaz AlabarT ${ }^{105}$ después de analizar los distintos argumentos esgrimidos a favor y en contra de la adopción de los riesgos de desarrollo declaran:

«Todas estas reflexiones hacen que nos planteemos que, a primera vista, al menos, pueda parecer extraño, que un país como España, no precisamente entre los de mayor potencia económica de Europa, haya optado por extender la responsabilidad del fabricante a este tipo de riesgos, cuando la mayor parte de los Estados de la Unión Europea han desistido de ello».

102 Texto de la Ponencia de 26 de enero de 1988 preparada por el profesor Rojo (Boletín de Información del Ministerio de Justicia n. ${ }^{\circ} 1.489$, pp. 1.928 y ss.).

103 2-12-1988, NO 11-03.

104 Ciertos partidos políticos se opusieron argumentando que el artículo 15.1(b) de la Directiva permitía a los Estados incluir o excluir «completamente» los riesgos de desarrollo, pero que no permitía hacerlo sólo en relación a ciertos productos.

105 Gómez LaPlazA, Díaz Alabart, «Responsabilidad civil por los daños causados por productos defectuosos», en Actualidad Civil, 1995, n. ${ }^{\circ}$ 25, p. 537. 
Sin embargo es muy probable que la solución adoptada por la Ley española no dé lugar a las consecuencias desastrosas para la industria farmacéutica y de alimentos apuntadas por un sector de la doctrina. En primer lugar, porque las empresas que con mayor probabilidad se verían afectadas por esta medida no tienen gran importancia en España, por ejemplo las empresas farmacéuticas; y en segundo lugar, porque un análisis del resto de las disposiciones de la Ley lleva a la conclusión de que no será fácil para los demandantes que sus reclamaciones prosperen (por ejemplo, límites temporales, pecuniarios, productos excluidos, etc. $)^{106}$.

\section{Análisis del artículo 6.1.e)}

El artículo 6.1.e) hace referencia a que el estado de los conocimientos científicos y técnicos no permita apreciar la existencia del defecto. Está claro que no nos encontramos ante una situación similar a la que se da en algunos Estados norteamericanos, en la que se discute la existencia o no del defecto. Evidentemente, el producto del que se trata en este artículo es defectuoso. Por ello es importante examinar cómo debe ser el estado de los conocimientos científicos y técnicos que permiten la causa de exclusión de responsabilidad recogida en el 6.1.e). El criterio, mayoritariamente aceptado, es la incapacidad de descubrir el defecto para todo el mundo. Estamos de acuerdo con PARRA LUCÁn cuando dice que la imposibilidad es absoluta e independiente de las dificultades que suponga la investigación precisa de la existencia del defecto e, incluso, del nivel de gastos que comporte ${ }^{107}$. Se ha discutido hasta qué punto la existencia, por ejemplo, de un informe médico elaborado en japonés puede evitar la aplicación de la exoneración de responsabilidad. Debido a la falta de jurisprudencia sobre esta cuestión nos es imposible aventurar una respuesta, aunque, si la norma se aplicase de forma estricta, la mera existencia de ese informe o artículo escrito en japonés debería ser suficiente para impedir la aplicación del artículo 6.1.e). Sin

106 De esta misma opinión son las autoras anteriores Gómez LAPLAZA y DíAz Alabart, op. cit., p. 537:

«En realidad, el problema no es en general tan grande como parece, y menos aún para el caso de España.»

107 V. María A. PARra LuCÁn, cit., p. 521. Debemos dejar constancia de que la profesora PARRA LuCÁn hace estas apreciaciones en su comentario sobre los riesgos de desarrollo en la Directiva. Sin embargo, consideramos que no hay inconveniente en trasladar estas afirmaciones al conocimiento que se exige en la Ley española porque ésta transcribe fielmente, en este apartado, el texto de la Directiva. 
embargo, no parece probable que los tribunales lleven a cabo una interpretación tan rigurosa del artículo.

El momento en el que se debe analizar el estado de los conocimientos científicos y técnicos existentes es el momento de «puesta en circulación del producto». Sin embargo, y aunque este concepto aparece abundantemente en el texto de la Ley ${ }^{108}$, su significado no es siempre sencillo porque ni la Directiva ni la Ley española lo definen. El Convenio del Consejo de Europa, en su artículo 2 d) ofrece una definición de «puesta en circulación». Dispone este artículo que un producto ha sido puesto en circulación cuando el productor lo ha entregado a otra perso$\mathrm{na}^{109}$. Otra característica que se desprende de la legislación comunitaria (en especial art. 7.a) de la Directiva y 6.1.a) de la Ley española) es la voluntariedad. Si el producto no ha sido puesto en circulación de forma voluntaria por el fabricante o productor, éste no responde. Además hay que tener en cuenta que los posibles responsables de una responsabilidad por productos defectuosos no son sólo los fabricantes del producto, sino que, por ejemplo, también lo pueden ser los importadores o los suministradores (art. 4 Ley española ${ }^{110}$ ) y ésta es la cuestión más difícil de responder, ya que el momento de puesta en circulación de cada uno de los posibles responsables no tiene por qué ser coincidente.

De acuerdo con la definición recogida más arriba del Consejo de Europa, el momento de puesta en circulación será para el importador

108 Por ejemplo, en la Exposición de Motivos «La responsabilidad objetiva del fabricante dura diez años desde la puesta en circulación del producto defectuoso causante del daño»; artículo 3.1, 3.3, artículo 6.1.a), artículo 6.1.b), artículo 13, y disposición transitoria única.

109 V. Alcover Garau, La responsabildiad civil del fabricante. Derecho comunitario y adaptación al Derecho español, Madrid, 1990, pp. 118 y ss.

110 Artículo 4. Concepto legal de fabricante e importador:

«1. A los efectos de esta Ley se entiende por fabricante:

a) El de un producto terminado.

b) El de cualquier elemento integrado en un producto terminado.

c) El que produce una materia prima.

d) Cualquier persona que se presente al público como fabricante, poniendo su nombre, denominación social, su marca o cualquier otro signo distintivo en el producto o en el envase, el envoltorio o cualquier otro elemento de protección o de presentación.

2. A los mismos efectos, se entiende por importador quien, en el ejercicio de su actividad empresarial, introduce un producto en la Unión Europea para su venta, arrendamiento, arrendamiento financiero o cualquier otra forma de distribución.

3. Si el fabricante no puede ser identificado, será considerado como fabricante quien hubiera suministrado o facilitado el producto, a menos que, dentro del plazo de tres meses, indique al dañado o perjudicado la identidad del fabricante o de quien le hubiera suministrado o facilitado a él dicho producto. La misma regla será de aplicación en el caso de un producto importado, si el producto no indica el nombre del importador, aun cuando se indique el nombre del fabricante.» 
cuando éste lo entregue voluntariamente a otra persona, lo mismo para el fabricante o para el suministrador si el productor no puede ser identificado ${ }^{111}$. Es decir, cuando cada uno de ellos pierde voluntariamente el control sobre el producto. Sin embargo, existen autores que consideran que ésta no es la solución adecuada. Por ejemplo, Alcover GARAU ${ }^{112}$ :

«Así, si se estima que el fabricante no debe responder por riesgos de desarrollo, parece ilógico por ejemplo que por conocerse éstos en el lapso de tiempo que media entre la puesta en circulación del producto del fabricante y la del importador deba responder éste que no tiene por qué conocerlos ni cómo controlarlos. Y lo mismo se puede decir del fabricante aparente.

Por tanto, se puede concluir que la puesta en circulación relevante a los efectos de la Directiva sólo puede ser la de los fabricantes y no la del resto de legitimados sean éstos directos o supletorios. Cualquier otra solución no parece aceptable, ya que grava la posición de los distribuidores cuando no hay razones para proteger a los consumidores frente a los fabricantes que son los auténticos responsables».

Nosotros no estamos de acuerdo con esta postura. La Directiva y la Ley española han contemplado una pluralidad de sujetos como posibles responsables de los daños ocasionados por un producto inspirados por el deseo de dispensar la máxima protección al consumidor (considerando cuarto del texto comunitario). Se busca garantizar la indemnización a la víctima del daño. Por ello si adoptamos la postura anterior estaríamos vaciando de contenido el artículo 4 de la Ley española y los motivos y fines que en él subyacen.

\section{Análisis del artículo 6.3}

España ha sido el único país de la Comunidad Europea que ha optado por excluir de la defensa de los riesgos del desarrollo a una serie de productos. La mayoría de los países ha declarado la defensa aplicable

111 De la misma opinión es la profesora PARRA LuCÁn, cit., p. 526:

«A mi juicio, y habida cuenta de que aparecen equiparados al productor una serie de sujetos la puesta en circulación del producto aparecerá referida para cada uno de ellos, al momento en que se desprenden voluntariamente del producto: la salida de fábrica, la entrega a otro sujeto de la cadena de distribución, la venta al consumidor final...».

112 Alcover Garau, cit., pp. 122-123. De la misma opinión es R. Bercovitz, La responsabilidad de los fabricantes en la Directiva... p. 122, citado en PARRA LuCÁN, op. cit., p. 529): «la carga de estar al corriente del conocimiento científico y técnico y de utilizarla en relación con los defectos de los productos sólo se puede imponer lógicamente al productor». 
para todos los productos dentro del ámbito de la Ley o han optado por no incorporar la defensa dentro del ámbito de aplicación de la Ley (por ejemplo, Luxemburgo).

En primer lugar debemos analizar cuáles son los productos para los que no se podrá invocar la causa de exoneración de la letra e) del apartado 1 del artículo 6: medicamentos, alimentos y productos alimentarios destinados al consumo humano. La ley de medicamentos española considera como medicamentos también a aquéllos destinados a animales o medicamentos veterinarios ${ }^{113}$. PARRA LUCÁN ${ }^{114}$ cree que existen razones para pensar que la exigencia de estar «destinados al consumo humano» puede ser también aplicable a los medicamentos y por tanto aquéllos destinados a animales quedarían fuera del ámbito de aplicación del artículo 6.3. Por el contrario, si están incluidos los medicamentos de origen humano (sangre, plasma) porque, usados con fines terapéuticos, son considerados como medicamentos (art. 40 de la Ley del medicamento). Con esta inclusión se separa la Ley española de las posturas mayoritariamente adoptadas en otros países, como por ejemplo en los Estados Unidos, en los que por los especiales beneficios que reporta la transfusión sanguínea se ha optado por excluir a éstos del ámbito de la responsabilidad objetiva ${ }^{115}$. Los artículos $1.02 .01 \mathrm{y}$ 1.02.12 del Decreto 2.484/1967, de 21 de setiembre, por el que se aprueba el Código alimentario español son de aplicación en lo que se refiere a los conceptos de «alimentos» y de «productos alimentarios».

El artículo 6.3 debe examinarse en relación con el límite total de la responsabilidad (art. 11), y la extinción de responsabilidad transcurridos 10 años desde la puesta en circulación del producto (art. 13).

113 Artículos 8, 43 y ss. de la Ley 25/1990, de 20 de diciembre.

114 V. PARRA LuCÁN, «Notas a la Ley 22/1994, de 6 de julio, de responsabilidad civil por los daños causados por productos defectuosos», en Actualidad Civil, 1995, n. ${ }^{\circ}$ 36, p. 746.

115 V. Gómez Laplaza, Díaz Alabart, op. cit., p. 539:

«(...) un segundo punto, podría ser una razón de ineludible coherencia del legislador español con su compromiso con la protección del consumidor. Como se ha puesto de relieve ya en la ponencia de la Dra. Gómez Laplaza, en ciertos extremos nuestra Ley de Consumidores, ofrecía mayor protección al consumidor que la consagrada en la Directiva comunitaria, y sin embargo con el sistema adoptado para la trasposición se ha renunciado parcialmente a ese mayor nivel de protección conseguida, en aras de una mayor uniformidad con los derechos europeos en sede de protección por productos defectuosos. Quiero creer que nuestro legislador, de acuerdo con el mandato del artículo $51 \mathrm{CE}$, no ha querido dejar sin una protección especial a los perjudicados por unos productos, que como los medicamentos y alimentos son tan susceptibles de causar daños personales. No podemos olvidar que el origen de nuestra Ley de Consumidores de 1984, es la conmoción social producida por un envenenamiento masivo producido por un producto alimentario, el aceite de colza desnaturalizado». 
Los daños causados por riesgos del desarrollo normalmente aparecen años después de que el producto haya sido puesto en circulación. En ocasiones el período transcurrido entre el descubrimiento científico o técnico que permite descubrir el daño y la puesta en circulación es superior al de 10 años (por ejemplo, los daños producidos por el asbesto). Según el artículo 13 de la Ley española los derechos reconocidos en dicha Ley se extinguirán transcurridos 10 años a contar desde la fecha en que se hubiera puesto en circulación el producto concreto causante del daño, a menos que, durante ese período, se hubiese iniciado la correspondiente reclamación judicial ${ }^{116}$. De la aplicación conjunta del artículo 6.3 y del límite del artículo 13 se llega a la conclusión de que un gran número de reclamaciones posibles que podían estar basadas en el artículo 6.3 (y por lo tanto el responsable no se eximiría de responsabilidad y la víctima del daño lograría la reparación), se verán malogradas por el transcurso del plazo de 10 años recogido en el artículo 13. Esta es probablemente una de las razones por las que la peculiaridad de la Ley española frente a la Directiva no dará lugar a grandes diferencias prácticas entre ellas, o entre la Ley española y aquellas leyes europeas que en la incorporación de la Directiva a su derecho interno han optado por incluir en su totalidad la excepción de responsabilidad de los riesgos de desarrollo ${ }^{117}$.

El artículo 11 de la Ley española recoge un límite en la cuantía de 10.500.000.000 de pesetas para la responsabilidad civil global del fabricante o importador por muerte y lesiones personales causadas por productos idénticos que presenten el mismo defecto ${ }^{118}$. Los daños causados por riesgos del desarrollo normalmente se sufren por un gran número de víctimas y los productos que han causado el daño son productos idénticos, con lo que claramente el límite económico es de aplicación a

116 El considerando número once de la Directiva indica que «no sería razonable hacer responsable al productor del estado defectuoso de su producto por tiempo ilimitado». V. artículo 11 de la Directiva:

«Los Estados miembros dispondrán en sus legislaciones que los derechos conferidos al perjudicado en aplicación de la presente Directiva se extinguirán transcurrido el plazo de diez años a partir de la fecha en que el productor hubiera puesto en circulación el producto mismo que causó el daño, a no ser que el perjudicado hubiera ejercitado una acción judicial contra el productor.»

117 De la misma opinión son Parra LuCÁn, cit., p. 746 y Gómez LAPLAZA, cit., p. 538.

118 Artículo 16 de la Directiva:

1. Cualquier Estado miembro podrá disponer que la responsabilidad global del productor por los daños que resulten de la muerte o lesiones corporales causados por artículos idénticos que presenten el mismo defecto, se limite a una cantidad que no podrá ser inferior a 70 millones de ECUS. 
los casos del 6.3 y es aquí, en el artículo 11, donde volvemos a encontrar un contrapeso a la responsabilidad objetiva «absoluta» recogida en el artículo 6.3.

Alcober Garau se pregunta:

«¿qué ocurrirá si una vez indemnizadas una gran serie de víctimas por un montante global de 40.000.000 de ECUS, aparecen otras víctimas que pretenden en conjunto una indemnización de 50.000 .000 de ECUS?» ${ }^{119}$

Este autor considera que el límite es elevado, «incluso demasiado para ser un límite», y además «en la medida en que los supuestos de riesgos de desarrollo son, excepto al parecer en el sector farmacéutico, muy poco frecuentes».

Es cierto que los daños causados por defectos desconocidos en el momento de puesta en circulación de un producto no son frecuentes, pero cuando se materializan el número de afectados suele ser de miles de personas, por ello no creemos que se pueda considerar que el límite sea «demasiado» elevado. El supuesto de AlCOBER GARAU — de distintas indemnizaciones que en conjunto superan el límite de la Ley- puede surgir en un sistema en el que no se recogen los riesgos del desarrollo como causa de exclusión de la responsabilidad. En estos casos creemos que se debería recoger algún método de acción colectiva (por ejemplo, algo similar a la class action norteamericana), puesto que de lo contrario nos podríamos encontrar con supuestos en los que debido al gran número de afectados y a la gravedad de las lesiones la suma de las indemnizaciones superase el límite legal. Podría ocurrir que aquellos que reclamasen primero tendrían probablemente mayor oportunidad de lograr la indemnización de sus daños que aquellos que reclaman una vez superado el límite, que no tendrán ninguna oportunidad de lograr indemnización en base a la Ley 22 .

Por último, la Directiva establece un procedimiento específico bastante complejo para el caso de que un Estado miembro quiera eliminar la causa de exoneración del fabricante de los riesgos del desarrollo (art. 15.1.b). En este precepto se señala la exigencia de que el Estado comunique a la Comisión el texto de la medida propuesta, a fin de que ésta informe de ello a los demás Estados, debiendo esperar nueve meses a partir de la notificación para adoptar la medida, pudiendo la Comisión presentar al

119 Alcover Garau se hace la pregunta en relación al límite recogido en la Directiva de 70 millones de ECUS. Consideramos que no existe problema alguno en utilizar la misma pregunta para el caso español ya que éste ha recogido casi literalmente lo dispuesto en este sentido en la Directiva. 
Consejo propuesta de modificar la Directiva, en cuyo caso el Estado miembro deberá esperar dieciocho meses. El Preámbulo de la Ley española mantiene silencio sobre esta cuestión y el Estado español no ha seguido dicho procedimiento. Dado el rango normativo superior de la Directiva respecto a la Ley interna, podría ser que la norma española en este aspecto deviniera ineficaz ${ }^{120}$.

\section{Sumario}

La Ley española, en primer lugar, transcribe casi literalmente la norma comunitaria sobre riesgos del desarrollo. Quedan sin solucionar, por su indefinición, la determinación del «momento de puesta en circulación» aunque en nuestra opinión éste debe ser cuando el producto abandona de forma voluntaria las manos del productor, importador o suministrador. La peculiaridad de la Ley española se encuentra en el artículo 6.3 en el que se excluye del régimen general de aplicación de la causa de exoneración de responsabilidad de los riesgos de desarrollo a los medicamentos, alimentos o productos alimentarios destinados al consumo humano. Lo que a primera vista parece una quiebra importante, no lo es tanto si se analiza junto con el resto de las normas de la Ley. El límite de 10 años y el límite económico suponen un importante contrapeso y tal vez tengan como efecto que el artículo 6.3 no tenga la importancia práctica que en un primer momento se le pudiera suponer. Es de lamentar que el Tribunal Supremo no haya tenido, por ahora, la oportunidad de analizar las cuestiones anteriores.

120 El «Primer informe subre la aplicación de la Directiva en materia de responsabilidad por los daños causados por productos defectuosos (85/374/CEE)» no ha hecho referencia alguna a esta cuestión, salvo la mención genérica de que los «riesgos de desarrollo»serán objeto de seguimiento. 


\section{ANEXO}

\section{Incorporación a las leyes nacionales de los Estados miembros 121}

Según el artículo 19 de la Directiva, los Estados miembros deberán adoptar las medidas necesarias para cumplirla en un plazo de tres años, como máximo, a partir del día de su notificación e información a la Comisión. El plazo concluyó el 30 de julio de 1988 y pocos Estados habían incorporado la Directiva a sus derechos internos (Austria, Grecia, Italia y Gran Bretaña).

\section{Alemania}

La responsabilidad por productos aparece por primera vez en Alemania en la decisión del Tribunal Supremo Federal de 26 de noviembre de $1968^{122}$. El Código civil alemán recoge la responsabilidad basada en culpa o negligencia en la sección 823 y ss. La ley de 1990 establece las bases legales adicionales para entablar una acción de productos defectuosos ${ }^{123}$.

Sección 1

(2) El fabricante no es responsable si:

5) el estado del conocimiento científico y técnico, en el momento en que el productor puso el producto en circulación, no permitía descubrir el defecto.

Sin embargo, si el defecto del producto es conocido una vez que éste se halla en el mercado y el productor no informa a los consumidores, el productor podrá ser declarado responsable bajo los principios tradicionales de responsabilidad.

La defensa de los riesgos del desarrollo no se aplica a los daños causados por productos farmacéuticos, los cuales entran dentro del ámbito de aplicación de la Ley de productos farmacéuticos (Arzneimittelgesetz) ${ }^{124}$

121 Para más información sobre las leyes europeas de responsabilidad del fabricante ver: AAVV, European Product Liability, London, 1992; AAVV, Product Liability. European Laws and Practice, London, 1993; Howells, G., Comparative Product Liability, Aldershot, 1993; AAVV, International product Liability, London, 1993.

122 BGH decisión de 26-11-1968 - VI ZR 212/66- NJW 1969, 384- «Hühnerpest». V. European Product Liability, cit., p. 125.

${ }^{123}$ La Ley de productos defectuosos entró en vigor el 1 de enero de 1990.

${ }^{124}$ El sistema especial de responsabilidad por fármacos defectuosos fue introducido en Alemania como consecuencia del caso Contagan (los fetos de mujeres embarazadas que habían utilizado el fármaco en cuestión, sufrieron graves lesiones físicas y psíquicas). 


\section{Austria}

La responsabilidad por incumplimiento contractual y extracontractual en Austria se encuentra recogida en el Código civil de 1811 125 . El 21 de enero de 1988 el Parlamento austríaco aprobó la Ley federal de responsabilidad por productos defectuosos $(\mathrm{PHG})^{126}$. La situación previa a la Ley de 1988 era la responsabilidad del productor por productos defectuosos basada: o en la relación contractual entre las partes o en la responsabilidad por culpa. El productor podía eximirse de responsabilidad con la prueba de que había adoptado todas las medidas necesarias. Además, sólo estaba protegido el usuario autorizado, no el «bystander» ${ }^{127}$.

El n. ${ }^{\circ} 2$ de la sección 8 del PHG permite al fabricante eximirse de responsabilidad si prueba que en el momento en que el producto se puso en circulación, habiéndose aplicado todos los conocimientos científicos y técnicos pertinentes, no se podía haber detectado en el producto en cuestión el defecto que causó la lesión. El momento de la distribución del producto es relevante porque el importador es responsable de aquellos defectos que sean descubiertos como consecuencia de los desarrollos técnicos durante el período de almacenamiento del producto.

\section{Bélgica}

Artículo 8 de la Ley de 25 de febrero de 1991: bar que:

El productor será responsable bajo esta Ley a menos que pueda pro-

e) el state of the art desde el punto de vista científico y técnico no le permitió, en el tiempo en que el producto fue puesto en el mercado, detectar la existencia del defecto;

La Ley belga no ha supuesto un cambio fundamental del sistema anterior, puesto que éste ya incluía conceptos de responsabilidad objetiva

125 Allgemeines Bürgerliches Gasetzbuch (ABGB).

126 Bundesgesetz vom 21. Jänner 1988 über die Haftung für ein fehlerhaftes Produkt (Produkthaftungsgesetz); Federal Law Gazette Nr. 99 of 1988 (Bundesgesetzblatt 99/1988). Aunque Austria no era un Estado miembro, la aprobación de la Ley de responsabilidad por productos defectuosos trató de solucionar ciertos problemas que la doctrina y los tribunales habían detectado en el sistema del Código civil y la creación de un mercado competitivo para sus productos dentro de Europa.

127 V VV.AA., European Product Liability, Butterworths, London, pp. 15 y ss., 1992. 
similares a los de la Directiva. Es cierto que Bélgica no había adoptado una legislación específica de protección del consumidor, ya que éste era un campo multidisciplinar con elementos de derecho civil, mercantil, penal y administrativo. Fueron los órganos jurisprudenciales los que con su interpretación de la ley existente habían evolucionado hacia una protección del consumidor y hacia conceptos similares a los de la Directiva.

\section{Dinamarca}

En Dinamarca la responsabilidad por actos ilícitos no había sido recogida en una ley escrita ${ }^{128}$, pero había sido desarrollada por la jurisprudencia, la cual normalmente aplicaba la teoría de la culpa a la responsabilidad por productos defectuosos. La Directiva fue incorporada al derecho interno casi literalmente, tan sólo con unas escasas modificaciones, en la Ley 371 de 7 de junio de 1989.

Ley 371 de 7 de junio de $1989^{129}$, parte 3 apartado 7 :

(1) El fabricante no será responsable si prueba que:

4) con el conocimiento científico y técnico disponible en el tiempo en que el producto fue puesto en el mercado, no era posible descubrir el defecto.

\section{Francia}

En Francia, la responsabilidad del fabricante por productos defectuosos tradicionalmente ha estado basada en las reglas de responsabilidad del Código civil. Los tribunales han trabajado para extender esta protección básica del consumidor. Por ejemplo, del artículo 1.384, párrafo 1 del Código civil, la jurisprudencia ha elaborado un principio de responsabilidad objetiva para los daños causados por productos que una persona tenga en su custodia.

Aunque la defensa de los riesgos del desarrollo no existía en el derecho francés, los productos farmacéuticos disfrutaban de un estatus

128 La Danish Sale of Goods Act de 1904 establecía específicamente que las reglas de responsabilidad por defectos en los bienes vendidos no eran aplicables a los daños por productos.

129 Lov nr. 371 af 7. juni af 1989 om produktansvar. 
particular. Los tribunales habían llegado a la conclusión de que un fabricante no podía ser declarado responsable por el daño causado por un producto farmacéutico siempre que éste fuese el único capaz de proporcionar un cierto tratamiento médico ${ }^{130}$. Sin embargo, cuando se pone en el mercado un producto competidor nuevo y este último es menos lesivo que el primero, el fabricante del primer producto debe retirarlo del mercado $^{131}$.

El Gobierno francés optó por la reforma del Código civil como forma de incorporar los principios de la Directiva a su derecho interno. El Proyecto de Ley de reforma del Código civil que se discutió el 11 de junio de 1992, de forma expresa, establecía que el productor podía ser declarado responsable aun cuando el producto y el fabricante cumpliesen con el state of the art o estuvieran sujetos a la autorización de un cuerpo administrativo (art. 1.386-9). La no adopción de la defensa de los riesgos del desarrollo era consecuente con la jurisprudencia francesa existente.

Cuando se elaboró el primer Proyecto de Directiva el Gobierno francés se opuso con fuerza a la defensa de los riesgos del desarrollo. El 19 de mayo de 1998 se aprobó la Ley relativa a la responsabilidad debida a productos defectuosos ${ }^{132}$, y en el nuevo artículo 1.386-11 (4) del Código civil francés se recoge la defensa de los riesgos del desarro1lo. Es decir, que la reforma no hace uso de la posibilidad recogida en el artículo 15.1(b) de la Directiva para mantener la ley existente. Se ha justificado la inclusión de la defensa de los riesgos del desarrollo en base a que así se evita la existencia de una desventaja competitiva para los productores franceses frente a aquellos productores de países pertenecientes a la Comunidad Europea que sí recogen la defensa. Dicha defensa no existía en el derecho francés anterior, por lo que para lograr que sea efectiva fue necesario eliminar la posibilidad de que una persona pudiese alegar los derechos previos basados en las reglas de responsabilidad contractual y extracontractual anteriores a la implementación de la Directiva.

Es probable que los tribunales franceses sean muy estrictos en su valoración del estado de los conocimientos científicos y técnicos que permitan eximir de responsabilidad al fabricante.

130 Cour de Cassation, 1 st Civil Chamber, 8 october 1970, Bull Civ I, no 248. V. European Product Liability, cit., p. 115.

131 Cour de Cassation, Civil Chamber, 25 may 1973, JCP 1975, II, 17955. V. European Product Liability, cit., p. 115.

${ }_{132}$ Loi no 98-389 du 19 mai 1998 relative à la responsabilité du fait des produits défectueux. 


\section{Grecia}

En Grecia, en un primer momento, se adoptó el Decreto Ministerial de 31 de marzo de 1988, que entraba en vigor el 30 de julio de 1988. Pero este Decreto Ministerial no fue aprobado por el Parlamento. En septiembre de 1991 se aprobó la Ley 1.961/1991. Con anterioridad, los daños por productos defectuosos estaban cubiertos por las normas y los principios del Código civil griego, artículo 330 y ss. para la responsabilidad contractual, y artículo 914 y ss. para la responsabilidad extracontractual, así como algunas provisiones específicas que regulan la venta de bienes o productos.

La sección 10 de la Ley 1961/1991 dice:

El productor no es responsable si puede probar:

(e) que, en el momento en el que el producto se puso en circulación, él no conocía o no existía posibilidad alguna de que pudiese conocer la presencia del defecto.

No existe referencia alguna al «estado de los conocimientos científicos y técnicos» y además, parece que la excepción favorece un estándar subjetivo de análisis de la conducta del productor. El texto del Decreto Ministerial de 1988 era fiel al texto de la Directiva ${ }^{133}$.

\section{Holanda}

La incorporación al derecho interno holandés de los principios de la Directiva se llevó a cabo en la Ley que se presentó al Parlamento el 11 de setiembre de 1986 (n. ${ }^{\circ}$ 19.636). La Ley fue aprobada en marzo de 1990 y el 1 de noviembre de 1990 entraron en vigor los nuevos artículos del Código civil holandés: 1.407a-1.407j.

Artículo 1.407a

El productor será responsable por el daño causado por un producto defectuoso a menos:

1.e. Que fuese imposible, en base al state of the art de la ciencia y de la tecnología, detectar la existencia del defecto en el momento en que el producto fue puesto en el mercado.

133 Artículo 7.

De acuerdo con la presente decisión, el productor no es responsable si:

e) cuando introdujo el producto en el mercado, el nivel de conocimiento técnico y científico no permitía verificar la existencia de esos defectos. 
El 11 de setiembre de 1991, los artículos 1.407(a) a 1.407(i) se incorporaron al Código civil como los artículos 185 a 193. El Parlamento holandés ha manifestado su opinión de que el artículo 185, sección 1 (e), en el que se recoge la defensa de los riesgos del desarrollo, sea interpretado de forma restringida. La defensa sólo tendrá éxito «para aquellos defectos imposibles de descubrir, aun con el escrutinio más intenso» ${ }^{134}$.

En el caso Nefabas v. Janssen, la Hoge Raad ${ }^{135}$ trató la cuestión del state of the art. En este caso un trabajador demandó a su empleador por los daños sufridos al haber contraído la enfermedad de asbestosis. El trabajador alegó que había contraído la enfermedad cuando trabajaba para Nefabas, una compañía que utilizaba el asbesto. Nefabas alegó que durante el período en que el trabajador contrajo la enfermedad (de 1950 a 1960) no era consciente de los peligros que el asbesto suponía para la salud de los trabajadores, es decir, Nefabas alegó la defensa del state of the art. La Hoge Raad declaró que el empleador tiene la obligación de investigar, lo que supone que debe analizar los peligros de los materiales que se utilizan en su empresa, consultar a expertos y mantenerse informado sobre los desarrollos científicos y tecnológicos que afecten a su actividad. Sin embargo, también declaró que la defensa del state of the art, puede eximir al empleador de responsabilidad siempre que fuese virtualmente imposible descubrir el peligro.

\section{Irlanda}

La responsabilidad extracontractual del fabricante evolucionó en Irlanda de forma similar a Inglaterra, siguiendo el caso Donoghue v. Stevenson $^{136}$. El 16 de diciembre de 1991 entró en vigor la Liability for Defective Products Act y en el artículo 6 (e) se recoge la defensa de los riesgos del desarrollo:

6. El productor no será responsable bajo esta Ley si prueba:

e) que el estado del conocimiento científico y técnico en el momento en que el producto se puso en circulación no permitía que la existencia del defecto fuese descubierto;

134 Nota 2 TK 19636 , no. 9, p. 8.

135 HR 6 de abril 1990, NJ 1990, 573.

136 (1932) AC 562. 


\section{Italia}

La responsabilidad por productos defectuosos se recoge en el Decreto 224 de 24 de mayo de 1988 que incorpora al derecho italiano la Directiva. Las bases de responsabilidad del vendedor por la venta de sus productos la encontramos también en el Código civil (arts. 1.490-1.495) y los principios generales de la responsabilidad extracontractual, en el artículo 2.043 del mismo código.

Decreto Presidencial 224 de 31 de marzo de 1988.

Artículo 6

1. Se excluye la responsabilidad:

e) cuando el conocimiento científico y técnico, en el momento en que el productor puso el producto en circulación, no era capaz de considerar el producto como defectuoso.

El artículo 6(e) del Decreto adopta una terminología que difiere parcialmente de la del artículo 7(e) de la Directiva. En opinión de algunos autores ${ }^{137}$, el Decreto italiano al hacer referencia al hecho de que el conocimiento científico y tecnológico no permita que el producto sea considerado defectuoso parece introducir elementos «subjetivos» en la valoración del conocimiento técnico y científico.

\section{Luxemburgo}

La responsabilidad por productos es una de las áreas del Código civil de Luxemburgo que ha sido modificada para responder a las necesidades modernas. La reforma más importante fue la de 1987 en orden a facilitar las acciones de los consumidores contra los vendedores profesionales ${ }^{138}$. El derecho de Luxemburgo posteriormente se completó con Loi relative à la responsabilité civile du fait des produits défectueux de 21 de abril de $1989^{139}$, la cual incorpora al derecho interno la Directiva.

137 V. European Product Liability, cit., p. 231.

138 Loi du 15 mai 1987 modifiant et complétant certains articles du Code civil et complétant la loi du 25 aout 1983 relative a la protection juridique du concommateur, Mém.A, 1987.

139 Loi relative à la responsabilité civile du fait des produits défectueux, Mém.A, 1 989, 522. 
Aunque no incluye una excepción de riesgos del desarrollo, no se debe excluir la posibilidad de que los tribunales de Luxemburgo sigan a la jurisprudencia francesa sobre daños causados por productos farmacéuticos y admitan que el fabricante no es responsable por los daños causados por un producto farmacéutico si éste era el único posible para un determinado tratamiento médico ${ }^{140}$.

\section{Noruega}

En Noruega la Ley de productos defectuosos de 23 de diciembre de 1988 (Produktansvarsloven, número 144) fue reformada por la Ley de 27 de noviembre de 1992 (número 112). La Ley no ha adoptado la defensa de los riesgos del desarrollo. La Ley dedica el capítulo 3 a los productos farmacéuticos y en él crea un seguro que beneficia a los dañados por fármacos sin necesidad de probar que hubo un culpable o si el fármaco era defectuoso, basta con probar la lesión y la relación de causalidad entre el fármaco y la lesión ${ }^{141}$. El sistema de seguro para fármacos establece compensación también en casos en los que la lesión ha sido causada por un riesgo de desarrollo.

Antes de que la Ley entrase en vigor, era dudoso si se podía considerar responsable a un productor si el daño era debido a un riesgo del desarrollo. En 1974 la cuestión llegó al Tribunal Supremo de Noruega. El demandante alegó que el fabricante era responsable por los efectos secundarios de un contraceptivo que había causado embolismo en una mujer. Lamentablemente, el Tribunal no contesta la cuestión básica, ya que consideró que no se había probado la relación de causalidad entre el contraceptivo y la muerte de la mujer ${ }^{142}$.

\section{Portugal}

En 1981 se aprobó la Ley de protección del consumidor (N. ${ }^{\circ}$ 29/81 de 22 de agosto) con la intención de satisfacer los requisitos constitucionales de protección del consumidor (Constitución portuguesa, art. 60.1). En 1989 el Decreto Ley N. ${ }^{\text {383/89 de }} 6$ de noviembre incorporó la Directiva al derecho interno portugués.

\footnotetext{
140 V. European Products Liability, cit., p. 256.

141 V. International Product Liability, op. cit., pp. 442 y ss.

142 Rt. 1974, p. 1.160.
} 
Decreto Ley N. ${ }^{\circ}$ 383/89 de 6 de noviembre de 1989.
Artículo 5
El productor no es responsable si demuestra que
e) el estado del conocimiento técnico y científico, en el momento en que el producto fue puesto en circulación, no hacía posible detectar la existencia de defectos.

\section{Reino Unido}

La normativa del Reino Unido en materia de productos defectuosos se ha tratado en el apartado IV.

\section{Suecia}

En junio de 1991 se presentó ante el Parlamento sueco una propuesta de Ley. La propuesta fue estudiada después de las elecciones de setiembre de 1991 y las enmiendas fueron aprobadas por el Parlamento. La propuesta inicial no contenía la defensa de los riesgos del desarrollo pero fue incluida en la Ley aprobada en diciembre de $1991^{143}$.

8.4 si prueba que, según los conocimientos científicos y técnicos en el momento en que el producto fue puesto en circulación, no era posible descubrir la falta de seguridad.

\section{Suiza}

No existe en Suiza ninguna ley sobre la responsabilidad del fabricante por los daños causados en el uso o consumo de sus productos. Los principios que rigen son las reglas tradicionales de responsabilidad contractual y extracontractual. En 1979 se dieron algunos intentos por introducir una ley de responsabilidad por productos que fueron abandonados rápidamente. La doctrina predominante sostiene que un fabricante no es responsable de los defectos de sus productos (por lo

143 Produktansvarslag, 1991/92: LU 14. 
menos no en base al art. 41 del Código de obligaciones) si el defecto no pudo ser descubierto por el state of the art, en el momento en que salió al comercio. Tan pronto como el defecto del producto sea descubierto, el fabricante está obligado a informar a los consumidores o a modificar el diseño del producto si el defecto se encontraba en él, la omisión de actuar por parte del fabricante puede dar lugar a responsabilidad. 